This item was submitted to Loughborough's Research Repository by the author.

Items in Figshare are protected by copyright, with all rights reserved, unless otherwise indicated.

\title{
Maximal scarring for eigenfunctions of quantum graphs
}

PLEASE CITE THE PUBLISHED VERSION

https://doi.org/10.1088/1361-6544/aad3fe

PUBLISHER

IOP Publishing $\odot$ IOP Publishing Ltd \& London Mathematical Society

VERSION

AM (Accepted Manuscript)

PUBLISHER STATEMENT

This is the accepted version of the following article: BERKOLAIKO, G. and WINN, B., 2018. Maximal scarring for eigenfunctions of quantum graphs. Nonlinearity, 31 (10), pp.4812-4850, which has been published in final form at https://doi.org/10.1088/1361-6544/aad3fe.

\section{LICENCE}

CC BY-NC-ND 4.0

\section{REPOSITORY RECORD}

Berkolaiko, G., and Brian Winn. 2018. "Maximal Scarring for Eigenfunctions of Quantum Graphs”. Loughborough University. https://hdl.handle.net/2134/34066. 


\title{
Maximal scarring for eigenfunctions of quantum graphs
}

\author{
G. Berkolaiko \\ Department of Mathematics, Texas A83M University, College Station, TX77843-3368, U.S.A.
}

B. Winn

Department of Mathematical Sciences, Loughborough University, Loughborough, LE11 3TU, U.K.

$25^{\text {th }}$ June 2018

\begin{abstract}
We prove the existence of scarred eigenstates for star graphs with scattering matrices at the central vertex which are either a Fourier transform matrix, or a matrix that prohibits back-scattering. We prove the existence of scars that are half-delocalised on a single bond. Moreover we show that the scarred states we construct are maximal in the sense that it is impossible to have quantum eigenfunctions with a significantly lower entropy than our examples.

These scarred eigenstates are on graphs that exhibit generic spectral statistics of random matrix type in the large graph limit, and, in contrast to other constructions, correspond to non-degenerate eigenvalues; they exist for almost all choices of lengths.
\end{abstract}

\section{Introduction}

The possibility of existence of scarred quantum eigenstates has been a mystery of quantum mechanics since the intriguing suggestion of Heller [1] that for a quantum Hamiltonian corresponding to a chaotic classical limit, subsequences of eigenfunctions can converge in the high energy limit to a measure supported on one-or-more short unstable periodic orbits. This is the "strong" notion of scarring, as compared to the phenomenon of weak scarring, in which states are averaged over energy windows that are semiclassically increasing in size and which is now well-understood $[2,3,4,5,6,7]$.

Mathematically rigorous constructions of quantum scars have been found in some model systems: for quantum cat maps [8], and for some families of quantum graphs $[9,10]$, the topic to which the present article is devoted. (See also $[11,12,13]$ for results pertaining to eigenfunctions which localise around other invariant structures.) In the opposite direction, in certain situations it has been rigorously proved that scars cannot exist. These are models for which the quantum unique ergodicity property holds $[14,15]$.

In one sense, the question of "perfect" scarring as strictly described above has also been settled for a broad class of systems. On compact Riemann surfaces whose geodesic flow is chaotic, the entropy of quantum limits of eigenfunctions has a strictly positive lower bound $[16,17,18]$. A limiting measure supported on a finite union of periodic orbits would have entropy zero, and is thus ruled-out. However, the possibility remains open of a quantum limit that has a positive proportion of its mass supported on a periodic orbit, with the 
remaining mass, say equi-distributed. For a surface of constant negative curvature the results of [17] put a lower bound of $\frac{1}{2}$ on the entropy of quantum limits, meaning that at most half the mass can be carried by periodic orbits. The scars constructed on cat maps in [8] are also half-localised on periodic orbits and half equi-distributed, which was proved to be the maximal amount of delocalisation possible in that context in [19].

The study of statistical properties of quantum eigenfunctions and eigenvalues, particularly when the underlying classical system is chaotic, is part of the field of quantum chaos. The study of Schrödinger operators on one-dimensional networks is a prominent part of this field, going by the name of 'quantum graphs' [20,21, 22, 23, 24, 25, 26, 27, 28, $29,30,31,9,32,33]$ (the list of references is highly-incomplete but gives a flavour of the subject). In this article we report on constructions of scarred eigenfunctions of quantum graphs, that in some cases exhibit maximal delocalisation. We defer a full introduction to the quantum graphs to section 2, mentioning here only the necessary facts to introduce our results.

A graph is a network of vertices and bonds on which waves propagate and are scattered in accordance with scattering matrices attached to each vertex. An eigenfunction is a standing wave, which may be completely described by $2 B$-dimensional vector $\boldsymbol{a}$ giving the complex amplitudes of the wave on each directed bond. We will be interested in subsequences of eigenvectors converging to a limit that shows localisation - significant enhanced amplitude on certain subsets of bonds.

Colin de Verdière [9] has studied scarred eigenvectors of quantum graphs with Kirchhoff scattering matrices at all vertices, and proved that for irrational and independent bond lengths there exist subsequences of eigenfunctions which fully localise onto simple paths that are either: closed paths, or connect two distinct vertices of degree one (the former was initially observed in [7], the latter may be seen as a significant generalisation of an earlier result [10] by the authors). In [9] a convergence result is proved that will be a key tool in our analyses (see theorem 2.1 below).

Our main results concern star graphs, but we will focus on alternative choices of boundary conditions. Star graphs are graphs with a single central vertex surrounded by $B$ outlying vertices (see figure 1 below). It has been known for some time $[34,10]$ that there exist subsequences of eigenfunctions that localise on a pair of bonds for quantum star graphs with irrational bond lengths and Kirchhoff boundary conditions at the central vertex. We study certain non-Kirchhoff boundary conditions that have attracted interest in the subject ${ }^{1}$ and prove that there exist subsequences of eigenfunctions that half-localise on a single bond.

By 'half-localise' we mean that there exist limits of eigenfunctions which are an equal superposition of a state with all mass equally-distributed to all bonds, and a state that is fully concentrated on a single bond. These eigenfunctions are maximally scarred, as measured by their entropy, a fact which we show in section 3 .

The boundary conditions that we consider for the star graphs are those given by the Fourier transform matrix, and equi-transmitting matrices [35]. Both kinds of matrices are unitary; Fourier transform matrices have all components with equal amplitude, and equi-transmitting matrices have all off-diagonal components having equal amplitudes and diagonal entries zero.

The $6 \times 6$ Fourier transform matrix and an example of a $6 \times 6$ equi-transmitting matrix

\footnotetext{
${ }^{1}$ Principally because, in constrast to Kirchhoff star graphs, they exhibit spectral statistics agreeing with Random Matrix Theory; see the discussion below.
} 
are

$$
\mathcal{F}_{6}=\frac{1}{\sqrt{6}}\left(\begin{array}{cccccc}
1 & 1 & 1 & 1 & 1 & 1 \\
1 & \omega & \omega^{2} & \omega^{3} & \omega^{4} & \omega^{5} \\
1 & \omega^{2} & \omega^{4} & 1 & \omega^{2} & \omega^{4} \\
1 & \omega^{3} & 1 & \omega^{3} & 1 & \omega^{3} \\
1 & \omega^{4} & \omega^{2} & 1 & \omega^{4} & \omega^{2} \\
1 & \omega^{5} & \omega^{4} & \omega^{3} & \omega^{2} & 1
\end{array}\right), \quad E_{6}=\frac{1}{\sqrt{5}}\left(\begin{array}{rrrrrr}
0 & 1 & 1 & 1 & 1 & 1 \\
1 & 0 & 1 & -1 & -1 & 1 \\
1 & 1 & 0 & 1 & -1 & -1 \\
1 & -1 & 1 & 0 & 1 & -1 \\
1 & -1 & -1 & 1 & 0 & 1 \\
1 & 1 & -1 & -1 & 1 & 0
\end{array}\right)
$$

with $\omega=\mathrm{e}^{\mathrm{i} \pi / 3}$. Fourier transform matrices exist in any size, but it is not known whether or not $n \times n$ equi-transmitting matrices exist for every $n>3$ (it is known that no $3 \times 3$ equi-transmitting matrix exists). For example the authors are unaware of the existence of a $7 \times 7$ equi-transmitting matrix.

Our motivation for considering star graphs quantised with these scattering matrices comes from a consideration of the statistics of eigenvalues. For a quantum system arising as the quantisation of a classically chaotic system, the Random Matrix conjecture [36, 37] states that generically the eigenvalues do not occur independently, but rather are correlated at scales of the size of the mean spacing in exactly the same way as the limit in large matrix size of eigenvalues of ensembles of random matrices. The appropriate ensemble is governed by broad properties of the system such as whether it exhibits time-reversal invariance.

In earlier cases where eigenfunctions of quantum systems are rigorously know to scar $[8$, 10], the corresponding spectral correlations are different from those predicted by Random Matrix Theory (see [38] and [22, 25]). This does not invalidate the Random Matrix conjecture; it simply makes those systems non-generic. Also, the construction in [8] makes essential use of the fact that the corresponding eigenspaces are highly degenerate, so the fact that our eigenstates are simple is notable.

Star graphs with Fourier transform scattering matrices and equi-transmitting scattering matrices are expected to give spectral statistics consistent with the predictions of Random Matrix Theory [39] as the size of the graph tends to infinity. Our results, together with those of [9] establish the existence of scarred eigenfunctions in systems where (in the limit of large graph size) the spectral statistics seem to be generic for chaotic systems.

Our results presented in sections $3-5$ involve analyses of rank-one perturbations of certain complex Hadamard matrices and complex conference matrices, which may be of independent interest.

\section{Quantum graphs}

We introduce, in a brief way, the main definitions and ideas of quantum graphs. For details not given here we refer to [40], the review articles [41, 39] and the seminal papers $[20,21]$ on quantum chaos on graphs.

A graph $\Gamma=(V, E)$ consists of a set of vertices $V$ and bonds (or edges) $E$ with bonds connecting pairs of vertices. Our results are for star graphs. These are graphs with $B$ bonds each connecting outlying vertices to a single central vertex (see figure 1).

We make a graph $\Gamma$ into a metric graph by associating a positive length $L_{b}$ to each bond $b \in E$. It is convenient to record the bond lengths in a vector $L=\left(L_{b}\right)_{b \in E} \in \mathbb{R}_{>}^{B}$, where $B$ is the number of bonds. In many applications, and indeed sometimes below, the bond lengths $L$ will be chosen to be irrational and linearly independent over $\mathbb{Q}$.

A metric graph is made into a quantum graph by one of two procedures. The first such 


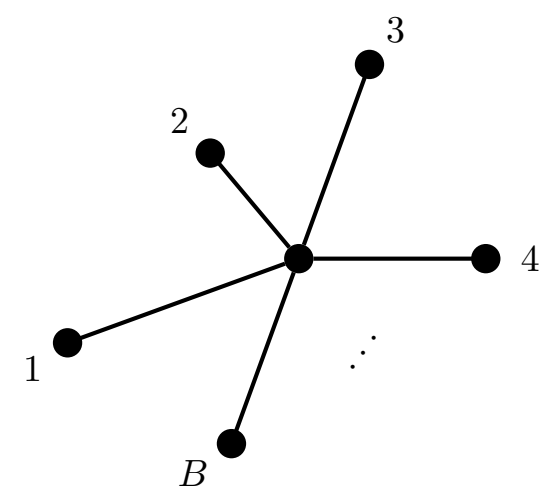

Figure 1: A star graph with $B$ bonds

procedure specifies a differential operator - usually of Schrödinger type - on the edges of the graph and matching conditions at the vertices. The second procedure, more popular in physical applications $[42,43,44]$, is to consider free wave propagation on the edges of the graph and scattering at the vertices. The two procedures are not entirely equivalent; an interested reader can find a comparison in [45]. We also note here that the second ("scattering" or "wave-dynamics") procedure is equivalent to the spectral problem for a first-order differential operator on a directified graph [46].

We will not describe the wave-dynamics model of quantum graphs in detail. The wave-function on a bond $b=(u, v)$ (here $u$ and $v$ are some vertices) is taken to be a superposition of two plain waves with momentum $k$, one propagating from $u$ to $v$ with the amplitude $a_{u, v}$ (as measured at $v$ ) and the other propagating from $v$ to $u$ with the amplitude $a_{v, u}$ (as measured at $u$ ),

$$
\psi_{u, v}(x)=a_{u, v} \mathrm{e}^{-\mathrm{i} k\left(L_{u, v}-x\right)}+a_{v, u} \mathrm{e}^{-\mathrm{i} k x} .
$$

It is convenient to index the amplitudes on the entire graph using $2 B$ directed bonds, denoted by $[u, v]$. A reversal operation is defined naturally on directed bonds by $\overline{[u, v]}=$ $[v, u]$.

Upon arrival at the vertex $v$, the wave is scattered into several waves coming out of $v$ and towards vertices $u^{\prime}$ adjacent to $v$. Some part of the wave may be reflected back to $u$; this is termed back-scattering. Conversely, a wave coming out of the vertex $v$ along the bond $[v, u]$ is the superposition of scattered waves which arrived at the vertex $v$ along bonds $\left[u^{\prime}, v\right]$. This process is described by a $d \times d$ unitary vertex scattering matrix $\sigma^{v}$ via the mapping

$$
a_{v, u} \leftrightarrow \mathrm{e}^{\mathrm{i} k L_{v, u}} \sum_{u^{\prime} \sim v} \sigma^{v}\left(u, u^{\prime}\right) a_{u^{\prime}, v},
$$

where $d$ is the degree of the vertex $v$. Here the exponential factor is the phase acquired by the wave while traversing the bond $[v, u]$ of length $L_{v, u}$. A value $k$ is an eigenvalue if there is a standing wave: the mapping (2.2) becomes an equality for each $[v, u]$.

An example of a scattering matrix for a vertex of degree $d$, is the (Neumann-)Kirchhoff scattering matrix

$$
\sigma_{\mathrm{N}}\left(u, u^{\prime}\right):=\frac{2}{d}-\delta_{u u^{\prime}}
$$

This particular scattering matrix may also be obtained through the first approach for the negative Laplacian with Kirchhoff (or "natural") vertex conditions. An advantage 
of the wave-dynamics approach is that one can specify scattering matrices that possess mathematically appealing properties, even if they do not arise out of boundary conditions for a self-adjoint operator, as was done in $[43,44,35]$ and as we will do below.

To record the scattering matrices for all vertices of a graph, it is typical to define a $2 B \times 2 B$ unitary bond scattering matrix $S$ with entries $S_{\left[v^{\prime}, u^{\prime}\right],[u, v]}$ indexed by directed bonds,

$$
S_{\left[v^{\prime}, u^{\prime}\right],[u, v]}:=\delta_{v v^{\prime}} \sigma^{v}\left(u^{\prime}, u\right) .
$$

The only non-zero entries of the matrix $S$ are the ones where $v^{\prime}=v$ and therefore the directed bond $\left[v^{\prime}, u^{\prime}\right]$ follows $[u, v]$ according to the connectivity of the graph.

The matrix $S$ is useful for more than just bookkeeping; it is the key to a powerful method of studying spectral properties of the graph which we now describe. Define the $2 B \times 2 B$ diagonal matrix $D=D(k)=D(k, L)$ by

$$
D_{\left[u^{\prime}, v^{\prime}\right],[u, v]}:=\delta_{u u^{\prime}} \delta_{v v^{\prime}} \mathrm{e}^{\mathrm{i} k L_{v, u}} .
$$

The condition for a standing wave with momentum $k$ (cf. (2.2)) takes the form

$$
\operatorname{det}(I-D(k) S)=0 .
$$

Assuming that $k=k_{n}$ is a value such that the determinant in (2.6) vanishes, there is at least one non-zero vector $\boldsymbol{a}=\left(a_{b}\right) \in \mathbb{C}^{2 B}$ such that

$$
\left(I-D\left(k_{n}\right) S\right) \boldsymbol{a}=0
$$

and the vector $\boldsymbol{a}$ is exactly the vector of amplitudes for the wave-function (2.1). We shall refer to vectors $\boldsymbol{a}$ satisfying (2.7) as quantum graph eigenvectors. It is common to call the product $U=D(k, L) S$ the quantum evolution operator for the graph, and $S$ the bond-scattering matrix (or $S$-matrix).

Note that writing down matrices $S$ in practice requires an ordering of the directed bonds. For star graphs we will always first list the bonds going to the central vertex (numbered by $j, j=1, \ldots, B$ ) and then list the bonds going in the opposite direction (numbered $j+B, j=1, \ldots, B$ ). Thus, the bond $j+B$ is the reversal of the bond $j$ and vice versa. From now on we will use this numbering in lieu of the directed bond notation $[u, v]$.

In order to prove existence of subsequences of eigenvectors converging to scarred states, we use the following result of Colin de Verdière:

Theorem 2.1. Consider a quantum graph with bond scattering matrix $S$. If $\boldsymbol{s} \in \mathbb{C}^{2 B}$, $\|\boldsymbol{s}\|=1$ is an eigenvector of $D\left(k_{0}, L_{0}\right) S$ for some $k_{0}$ and some set of bond lengths $L_{0}$, with a simple eigenvalue, then for any choice of incommensurate bond lengths $L$, there is a subsequence $\left(k_{n_{j}}\right) \subseteq\left(k_{n}\right)$ such that there exist normalised quantum graph eigenvectors $\boldsymbol{a}_{n_{j}}$ with eigenvalue $k_{n_{j}}$ that satisfy

$$
\boldsymbol{a}_{n_{j}} \rightarrow \boldsymbol{s} \quad \text { as } j \rightarrow \infty \text {. }
$$

Proof. This is basically part 2 of theorem 2.1 of [9], re-written in the language of scattering matrices. Note that Kirchhoff boundary conditions are assumed in the statement of theorem 2.1 of [9], but part 3 thereof is the only place where that assumption is necessary. Strictly speaking, the quoted result requires $s$ to be an eigenvector of the quantum graph, i.e. an eigenvector of $D\left(k_{0}, L_{0}\right) S$ with eigenvalue 1 , but it is easy to see that if there exists 
a choice of bond lengths $L_{0}$ for which $s$ has any other eigenvalue, by adjusting all lengths by the same amount we find an $L_{0}^{\prime}$ for which the eigenvalue is indeed 1 .

The main difficulty in applying theorem 2.1 will be to prove that the eigenvalue of $D\left(k_{0}, L_{0}\right) S$ is simple. In the cases that we consider we will do this by perturbing the bond lengths in an appropriate way.

For the sake of concreteness, we impose Kirchhoff conditions at the external vertices (leaves) of the star graphs, although other conditions could be specified there without substantially changing the results. At the central vertex, we choose scattering matrices that are either a Fourier transform matrix, or an equi-transmitting matrix.

To measure the degree to which limits of eigenvectors are scarred we will adopt the entropy measure as proposed on quantum graphs in [33].

Define the Shannon entropy $\mathrm{S}: \mathbb{C}^{2 B} \rightarrow[0, \log 2 B]$ by

$$
\mathrm{S}(\boldsymbol{a}):=-\sum_{b=1}^{2 B} \frac{\left|a_{b}\right|^{2}}{\|\boldsymbol{a}\|^{2}} \log \left(\frac{\left|a_{b}\right|^{2}}{\|\boldsymbol{a}\|^{2}}\right),
$$

and the Rényi entropy $\mathrm{R}_{\rho}: \mathbb{C}^{2 B} \rightarrow[0, \log 2 B]$ by

$$
\mathrm{R}_{\rho}(\boldsymbol{a}):=-\frac{1}{\rho} \log \left(\sum_{b=1}^{2 B}\left(\frac{\left|a_{b}\right|^{2}}{\|\boldsymbol{a}\|^{2}}\right)^{1+\rho}\right),
$$

for a parameter $\rho>-1, \rho \neq 0$. We also define

$$
\mathrm{R}_{\infty}(\boldsymbol{a}):=-\log \left(\max _{1 \leqslant b \leqslant 2 B}\left\{\frac{\left|a_{b}\right|^{2}}{\|\boldsymbol{a}\|^{2}}\right\}\right)
$$

which agrees with taking the limit $\rho \rightarrow \infty$ in (2.10). The Rényi entropy generalises the Shannon entropy in the sense that for $\boldsymbol{a} \in \mathbb{C}^{2 B}$,

$$
\lim _{\rho \rightarrow 0} \mathrm{R}_{\rho}(\boldsymbol{a})=\mathrm{S}(\boldsymbol{a})
$$

For a completely delocalised vector $\boldsymbol{a}=\frac{1}{\sqrt{2 B}}(1, \ldots, 1)$ we have $\mathrm{S}(\boldsymbol{a})=\mathrm{R}_{\rho}(\boldsymbol{a})=\log 2 B$, whereas for an eigenvector localised on one single bond, $\mathrm{S}(\boldsymbol{a})=\mathrm{R}_{\rho}(\boldsymbol{a})=\log 2$, so, roughlyspeaking, the smaller the entropy, the greater the measure of localisation, or strength of the scarring. On the other hand, the entropic uncertainty principle [47] places a theoretical lower-bound on the entropy of eigenstates. In section 3.2 we demonstrate that the entropic uncertainty theorem takes the following form.

Theorem 2.2. If $\boldsymbol{a}$ is any eigenvector of a Fourier transform star graph, the entropy of a satisfies

$$
\mathrm{S}(\boldsymbol{a}) \geqslant \frac{1}{2} \log B+\log 2 \quad \text { and } \quad \mathrm{R}_{\sigma /(1-\sigma)}(\boldsymbol{a})+\mathrm{R}_{-\sigma /(1+\sigma)}(\boldsymbol{a}) \geqslant \log B+2 \log 2,
$$

for any $0 \leqslant \sigma \leqslant 1$. If $\boldsymbol{a}$ is any eigenvector of an equi-transmitting star graph then

$$
\mathrm{S}(\boldsymbol{a}) \geqslant \frac{1}{2} \log (B-1)+\log 2 \quad \text { and } \quad \mathrm{R}_{\sigma /(1-\sigma)}(\boldsymbol{a})+\mathrm{R}_{-\sigma /(1+\sigma)}(\boldsymbol{a}) \geqslant \log (B-1)+2 \log 2 .
$$

We find it notable that the bound (2.13) is the average of the maximum and minimum possible entropies for graph eigenvectors.

In our results on star graphs in section 3 and section 5 we establish the lower-bounds and prove the existence of limiting eigenvectors that essentially achieve the bounds, i.e. maximally scarred states. 
Theorem 2.3. Consider a star graph with $B$ bonds, a Fourier transform scattering matrix at the central vertex, and incommensurate bond lengths. Then there exists a subsequence $\left(k_{n_{j}}\right) \subseteq\left(k_{n}\right)$ such that the corresponding normalised eigenvectors $\boldsymbol{a}_{n_{j}}$ satisfy

$$
\boldsymbol{a}_{n_{j}} \rightarrow \boldsymbol{a}^{\mathcal{F}}
$$

as $j \rightarrow \infty$, where $\boldsymbol{a}^{\mathcal{F}}$ is a vector with Shannon entropy

$$
\mathrm{S}\left(\boldsymbol{a}^{\mathcal{F}}\right)=\frac{1}{2} \log B+2 \log 2+\mathrm{O}\left(\frac{\log B}{B^{1 / 2}}\right)
$$

and the Rényi entropy is

$$
\mathrm{R}_{\infty}\left(\boldsymbol{a}^{\mathcal{F}}\right)+\mathrm{R}_{-1 / 2}\left(\boldsymbol{a}^{\mathcal{F}}\right)=\log B+2 \log 2+\mathrm{O}\left(B^{-1 / 2}\right) .
$$

For equi-transmitting star graphs we prove the following result in section 5 .

Theorem 2.4. Consider a star graph with $B$ bonds, an equi-transmitting scattering matrix at the central vertex, and incommensurate bond lengths. Then there is a subsequence $\left(k_{n_{j}}\right) \subseteq\left(k_{n}\right)$ such that the corresponding normalised eigenvectors $\boldsymbol{a}_{n_{j}}$ satisfy

$$
\boldsymbol{a}_{n_{j}} \rightarrow \boldsymbol{a}^{E}
$$

as $j \rightarrow \infty$, where

$$
\mathrm{S}\left(\boldsymbol{a}^{E}\right)=\frac{1}{2} \log (B-1)+2 \log 2,
$$

and

$$
\mathrm{R}_{\infty}\left(\boldsymbol{a}^{E}\right)+\mathrm{R}_{-1 / 2}\left(\boldsymbol{a}^{E}\right)=\log (B-1)+2 \log 2+\mathrm{O}\left(B^{-1 / 2}\right) .
$$

The Shannon entropy (2.9) was studied for quantum graphs in [33]. The authors used the entropic uncertainty principle to prove lower bounds for the entropy of eigenfunctions for regular graphs with equi-transmitting boundary conditions, and for equi-transmitting star graphs. They also calculate the average value of the entropy of star graphs with a Kirchhoff scattering matrix. Several of the results of [33] were repeated for the Rényi entropy in [48] as well as for a different generalisation of the Shannon entropy (the Tsallis entropy) that we do not consider here.

To compare the entropy bounds for existing eigenfunction localisations in the literature, the limiting eigenvectors $\boldsymbol{a}^{\mathrm{N}}$ of the star graph with Kirchhoff scattering matrices in [10] are localised equally on two bonds of the graph, and zero mass elsewhere, so the entropies are

$$
\mathrm{S}\left(\boldsymbol{a}^{\mathrm{N}}\right)=\mathrm{R}_{\rho}\left(\boldsymbol{a}^{\mathrm{N}}\right)=2 \log 2
$$

while the entropic uncertainty principle furnishes the lower bound

$$
\mathrm{S}(\boldsymbol{a}) \geqslant \log 2+\mathrm{O}\left(B^{-1}\right)
$$

and

$$
\mathrm{R}_{\sigma /(1-\sigma)}(\boldsymbol{a})+\mathrm{R}_{-\sigma /(1+\sigma)}(\boldsymbol{a}) \geqslant 2 \log 2+\mathrm{O}\left(B^{-1}\right)
$$

for eigenvectors of Kirchhoff star graphs.

The bounds in theorem 2.2, and theorem 2.3 are proved in section 3. The vectors $\boldsymbol{a}^{\mathcal{F}}$ in (2.15) have approximately half the mass concentrated on the first bond of the graph, and the remaining mass equidistributed. In section 4 we add further explanation about the process behind theorem 2.3, and explain how to construct eigenfunctions (half) localised on other bonds of the graph, and calculate their entropies. Some of the calculations from sections 3 and 4 have been placed in appendices. 


\section{$3 \quad$ Star graphs with non-Kirchhoff scattering matrices}

For a star graph with $B$ bonds, scattering matrix $\Sigma$ at the central vertex and Kirchhoff conditions at the external vertices, the $2 B \times 2 B$ bond scattering matrix is (see appendix A)

$$
S:=\left(\begin{array}{cc}
0 & \Sigma \\
I & 0
\end{array}\right)
$$

We are going to consider the two cases of $\Sigma$ being a $B \times B$ Fourier transform matrix, and a $B \times B$ equi-transmitting matrix. In contrast to the well-studied case where the central vertex has Kirchhoff boundary conditions, the Fourier transform and equi-transmitting cases are expected to produce generic spectral statistics in the limit $B \rightarrow \infty$.

\subsection{Sketch of the main ideas}

The $B \times B$ Fourier transform matrix $\mathcal{F}_{B}$ is defined to be the matrix with $j k$ th entry equal to

$$
\frac{1}{\sqrt{B}} \mathrm{e}^{2 \pi \mathrm{i}(j-1)(k-1) / B}
$$

The matrix $\mathcal{F}_{B}$ is unitary.

Equi-transmitting matrices were introduced in [35] as scattering matrices of quantum graphs. They are very closely related to complex conference matrices studied in the combinatorics literature. An $B \times B$ complex matrix $E$ is equi-transmitting if it is unitary with diagonal entries 0 and all other entries having equal absolute value.

As multiplication by a row or column by a pure phase does not change the equitransmitting property, we will assume that the equi-transmitting matrices used are of the form

$$
E_{B}=\frac{1}{\sqrt{B-1}}\left(\begin{array}{ccccc}
0 & 1 & 1 & \cdots & 1 \\
1 & 0 & * & \cdots & * \\
1 & * & 0 & \cdots & * \\
\vdots & \vdots & \vdots & \ddots & \vdots \\
1 & * & * & \cdots & 0
\end{array}\right)
$$

however our method would work with only minor changes for an arbitrary equi-trasmitting matrix, provided that its first row is identical to the transpose of the first column.

We define three vectors belonging to $\mathbb{C}^{B}$ :

$$
\boldsymbol{e}:=\frac{1}{\sqrt{B}}\left(\begin{array}{c}
1 \\
1 \\
\vdots \\
1
\end{array}\right), \quad \boldsymbol{u}_{1}:=\left(\begin{array}{c}
1 \\
0 \\
\vdots \\
0
\end{array}\right), \quad \tilde{\boldsymbol{u}}_{1}:=\frac{1}{\sqrt{B-1}}\left(\begin{array}{c}
0 \\
1 \\
\vdots \\
1
\end{array}\right)
$$

It is relatively easy to see that

$$
\mathcal{F}_{B} \boldsymbol{e}=\boldsymbol{u}_{1} \quad \text { and } \quad \mathcal{F}_{B} \boldsymbol{u}_{1}=\boldsymbol{e} .
$$

It therefore follows that the vectors

$$
e \pm \boldsymbol{u}_{1}
$$


are eigenvectors of $\mathcal{F}_{B}$ with eigenvalue \pm 1 , which moreover are strongly enhanced in the first component by a factor roughly $\sqrt{B}$. The Shannon entropy of $\boldsymbol{e} \pm \boldsymbol{u}_{1}$ is

$$
\frac{1}{2} \log B+\log 2 \mp \frac{1}{B^{1 / 2}} \log \left(B^{1 / 2} \pm 1\right) .
$$

Starting from this point we may construct eigenvectors of the scattering matrix of a $B$ bond Fourier star graph that are strongly localised on the first bond by a factor about $\sqrt{B}$.

The chief difficulty will be the fact that the eigenvectors $\boldsymbol{e} \pm \boldsymbol{u}_{1}$ belong to degenerate eigenspaces. Indeed the matrix $\mathcal{F}_{B}$ has eigenvalues $\pm 1, \pm \mathrm{i}$ with the dimensions of the corresponding eigenspaces approximately $B / 4$ for each eigenvalue $[49,50]$. This degeneracy means that we cannot immediately conclude that a subsequence of eigenfunctions converges to the localised state.

By a careful choice of bond lengths, we are able to resolve this degeneracy, and indeed construct highly-localised eigenvectors.

The case of equi-transmitting scattering matrices at the centre of the star graph proceeds similarly. In this case, the pertinent results (which may be easily checked) are

$$
E_{B} \boldsymbol{u}_{1}=\tilde{\boldsymbol{u}}_{1} \quad \text { and } \quad E_{B} \tilde{\boldsymbol{u}}_{1}=\boldsymbol{u}_{1}
$$

so that the localised eigenvectors of $E_{B}$ are

$$
\boldsymbol{u}_{1} \pm \tilde{\boldsymbol{u}}_{1}
$$

In fact, because $\boldsymbol{u}_{1}$ and $\tilde{\boldsymbol{u}}_{1}$ are orthogonal, the results for equi-transmitting matrices lead to comparatively cleaner formulæ (compare (3.33) with (5.2) below, for example).

\subsection{Some spectral facts}

The fact that we deal with $2 B \times 2 B$ scattering matrices of the form (3.1) rather than the unitary $B \times B$ matrices $\mathcal{F}_{B}$ or $E_{B}$ themselves will not be a major technical hurdle. The following standard result allows us to relate eigenvectors of the central scattering matrix with the full quantum evolution operator matrix.

Lemma 3.1. Suppose that $\Pi$ and $\Sigma$ are $B \times B$ matrices and that $\boldsymbol{u} \in \mathbb{C}^{B}$ is an eigenvector of $\Sigma \Pi$ with eigenvalue $\nu \neq 0$. Then the vectors

$$
\left(\begin{array}{c} 
\pm \nu^{1 / 2} \boldsymbol{u} \\
\Pi \boldsymbol{u}
\end{array}\right) \in \mathbb{C}^{2 B}
$$

where $\nu^{1 / 2}$ is some square root of $\nu$, are eigenvectors of the $2 B \times 2 B$ matrix

$$
T:=\left(\begin{array}{cc}
0 & \Sigma \\
\Pi & 0
\end{array}\right)
$$

with eigenvalues $\pm \nu^{1 / 2}$. Moreover, all eigenvectors of $T$ with eigenvalue different from 0 are of the form (3.10).

We may remark that if $\nu \neq 0$ the vectors (3.10) are linearly independent. 
Proof. Clearly the vectors (3.10) are non-zero, so we proceed by direct calculation:

$$
\begin{aligned}
\left(\begin{array}{cc}
0 & \Sigma \\
\Pi & 0
\end{array}\right)\left(\begin{array}{c} 
\pm \nu^{1 / 2} \boldsymbol{u} \\
\Pi \boldsymbol{u}
\end{array}\right) & =\left(\begin{array}{c}
\Sigma \Pi \boldsymbol{u} \\
\pm \nu^{1 / 2} \Pi \boldsymbol{u}
\end{array}\right) \\
& =\left(\begin{array}{c}
\nu \boldsymbol{u} \\
\pm \nu^{1 / 2} \Pi \boldsymbol{u}
\end{array}\right) \\
& = \pm \nu^{1 / 2}\left(\begin{array}{c} 
\pm \nu^{1 / 2} \boldsymbol{u} \\
\Pi \boldsymbol{u}
\end{array}\right)
\end{aligned}
$$

proving the first assertion. Contrariwise, suppose that $\boldsymbol{v}=\left(\begin{array}{l}\boldsymbol{x} \\ \boldsymbol{y}\end{array}\right)$ is an eigenvector of $T$ with eigenvalue $\lambda \neq 0$. From $T \boldsymbol{v}=\lambda \boldsymbol{v}$ and $T^{2} \boldsymbol{v}=\lambda^{2} \boldsymbol{v}$, we conclude that

$$
\boldsymbol{y}=\frac{1}{\lambda} \Pi \boldsymbol{x} \quad \text { and } \quad \Sigma \Pi \boldsymbol{x}=\lambda^{2} \boldsymbol{x},
$$

so $\boldsymbol{x}$ is an eigenvector of $\Sigma \Pi$ and

$$
\boldsymbol{v}=\frac{1}{\lambda}\left(\begin{array}{c}
\lambda \boldsymbol{x} \\
\Pi \boldsymbol{x}
\end{array}\right)
$$

which is of the form (3.10) up to an (irrelevant) scalar multiple.

We want to find a minimum theoretical bound for the entropy of eigenfunctions of star graphs with Fourier transform and equi-transmitting scattering matrices. A standard tool to accomplish this is the Entropic Uncertainty Principle, conjectured by Kraus [51] and proved by Maassen and Uffink [47]. Recall that two notions of the entropy of $\boldsymbol{a}$, were defined in (2.9) and (2.10) as

$$
\mathrm{S}(\boldsymbol{a}):=-\sum_{b=1}^{2 B} \frac{\left|a_{b}\right|^{2}}{\|\boldsymbol{a}\|^{2}} \log \left(\frac{\left|a_{b}\right|^{2}}{\|\boldsymbol{a}\|^{2}}\right) ; \quad \mathrm{R}_{\rho}(\boldsymbol{a}):=-\frac{1}{\rho} \log \left(\sum_{b=1}^{2 B}\left(\frac{\left|a_{b}\right|^{2}}{\|\boldsymbol{a}\|^{2}}\right)^{1+\rho}\right) .
$$

Theorem 3.2. ([47]) Let $U=\left(U_{i j}\right)$ be a unitary matrix. For any complex vector $\boldsymbol{v}$,

$$
\mathrm{S}(\boldsymbol{v})+\mathrm{S}(U \boldsymbol{v}) \geqslant-\log \left(\max _{i, j}\left|U_{i j}\right|^{2}\right)
$$

and for any $0 \leqslant \sigma \leqslant 1$,

$$
\mathrm{R}_{\sigma /(1-\sigma)}(\boldsymbol{v})+\mathrm{R}_{-\sigma /(1+\sigma)}(U \boldsymbol{v}) \geqslant-\log \left(\max _{i, j}\left|U_{i j}\right|^{2}\right) .
$$

If $\boldsymbol{v}$ is an eigenvector of $U$, then

$$
\mathrm{S}(\boldsymbol{v}) \geqslant-\frac{1}{2} \log \left(\max _{i, j}\left|U_{i j}\right|^{2}\right)
$$

and

$$
\mathrm{R}_{\sigma /(1-\sigma)}(\boldsymbol{v})+\mathrm{R}_{-\sigma /(1+\sigma)}(\boldsymbol{v}) \geqslant-\log \left(\max _{i, j}\left|U_{i j}\right|^{2}\right) .
$$


We remark that (3.16) and (3.18) may be obtained as the limit $\sigma \rightarrow 0$ of (3.17) and (3.19) respectively.

For a star graph the quantum evolution operator is of the form

$$
U=\left(\begin{array}{cc}
0 & \mathrm{e}^{\mathrm{i} k L} \Sigma \\
\mathrm{e}^{\mathrm{i} k L} & 0
\end{array}\right), \quad \mathrm{e}^{\mathrm{i} k L}:=\operatorname{diag}\left\{\mathrm{e}^{\mathrm{i} k L_{1}}, \ldots, \mathrm{e}^{\mathrm{i} k L_{B}}\right\},
$$

so it will not help to apply theorem 3.2 to $U$ directly as the entries of $U$ of absolute value 1 will produce a zero in the right-hand side of (3.18). We could apply the theorem to the matrix $U^{2}$ to get a non-trivial result; however we can do better. We note from the preceding discussion that eigenvectors of $U$ are of the form

$$
\boldsymbol{a}=\left(\begin{array}{c} 
\pm \nu^{1 / 2} \boldsymbol{x} \\
\mathrm{e}^{\mathrm{i} k L} \boldsymbol{x}
\end{array}\right)
$$

where $\boldsymbol{x}$ is an eigenvector of the unitary matrix $\mathrm{e}^{\mathrm{i} k L} \Sigma \mathrm{e}^{\mathrm{i} k L}$, with eigenvalue $\nu \in \mathbb{T}$.

We define entropies of $\boldsymbol{x} \in \mathbb{C}^{B}$ by

$$
\mathbf{s}(\boldsymbol{x}):=-\sum_{j=1}^{B} \frac{\left|x_{j}\right|^{2}}{\|\boldsymbol{x}\|^{2}} \log \left(\frac{\left|x_{j}\right|^{2}}{\|\boldsymbol{x}\|^{2}}\right) \quad \text { and } \quad \mathbf{r}_{\rho}(\boldsymbol{x}):=-\frac{1}{\rho} \log \left(\sum_{j=1}^{B}\left(\frac{\left|x_{j}\right|^{2}}{\|\boldsymbol{x}\|^{2}}\right)^{1+\rho}\right),
$$

Since the absolute value of the $j$ th entry of $\boldsymbol{a}$ of (3.21) is the same as the $(j+B)$ th entry, and (for that very reason)

$$
\|\boldsymbol{a}\|^{2}=2\|\boldsymbol{x}\|^{2}
$$

we have

$$
\mathrm{R}_{\rho}(\boldsymbol{a})=-\frac{1}{\rho} \log \left(2 \sum_{j=1}^{B}\left(\frac{\left|x_{j}\right|^{2}}{2\|\boldsymbol{x}\|^{2}}\right)^{1+\rho}\right)=\mathrm{r}_{\rho}(\boldsymbol{x})+\log 2 .
$$

and by taking the limit $\rho \rightarrow 0$, or by direct calculation,

$$
\mathrm{S}(\boldsymbol{a})=\mathrm{s}(\boldsymbol{x})+\log 2 .
$$

A related equality was noted in [33] (c.f. their Lemma 3).

Proof of theorem 2.2. We apply theorem 3.2 to the vectors $\boldsymbol{x}$ and the entropy (3.22). Since vector $\boldsymbol{x}$ is an eigenvector of $\mathrm{e}^{\mathrm{i} k L} \Sigma \mathrm{e}^{\mathrm{i} k L}$, and $\Sigma$ has bounded entries in the cases in which we are interested, we get

$$
\mathbf{s}(\boldsymbol{x}) \geqslant \frac{1}{2} \log B \quad \text { and } \quad \mathbf{r}_{\sigma /(1-\sigma)}(\boldsymbol{v})+\mathbf{r}_{-\sigma /(1+\sigma)}(\boldsymbol{v}) \geqslant \log B,
$$

if $\Sigma$ is a $B \times B$ Fourier transform matrix and

$$
\mathbf{s}(\boldsymbol{x}) \geqslant \frac{1}{2} \log (B-1) \quad \text { and } \quad \mathbf{r}_{\sigma /(1-\sigma)}(\boldsymbol{v})+\mathbf{r}_{-\sigma /(1+\sigma)}(\boldsymbol{v}) \geqslant \log (B-1),
$$

if $\Sigma$ is a $B \times B$ equi-transmitting matrix. These lead via (3.25) and (3.24) to entropy bounds (2.13) and (2.14) respectively.

A bound similar to (2.14) for equi-transmitting star graphs was derived by this method in [33]. In the following sections we construct eigenfunctions that meet these bounds to leading order. 


\subsection{Fourier scattering matrices}

We consider matrices of the form

$$
U_{\mathcal{F}}:=U_{\mathcal{F}}(\kappa):=\left(\begin{array}{cc}
0 & P(\kappa) \mathcal{F}_{B} \\
P(\kappa) & 0
\end{array}\right),
$$

where $P(\kappa):=\operatorname{diag}\left\{\mathrm{e}^{\mathrm{i} \kappa}, 1, \ldots, 1\right\}$. If we choose $k_{0}$ and $L_{0}$ so that $P(\kappa)=D\left(k_{0}, L_{0}\right)$, which can always be done, then (3.28) is the quantum evolution operator of a Fourier transform star graph with bond lengths $L_{0}$.

By lemma 3.1 eigenvectors of $U_{\mathcal{F}}$ are of the form

$$
\boldsymbol{a}=\left(\begin{array}{c} 
\pm \lambda^{1 / 2} \boldsymbol{x} \\
P(\kappa) \boldsymbol{x}
\end{array}\right)
$$

where $\boldsymbol{x}$ is an eigenvector of the matrix $P(\kappa) \mathcal{F}_{B} P(\kappa)$ with eigenvalue $\lambda \in \mathbb{T}$.

In a calculation that is deferred to appendix $\mathrm{B}$, we show that there are simple eigenvectors $\boldsymbol{x}_{ \pm}$of $P(\kappa) \mathcal{F}_{B} P(\kappa)$ with the form

$$
\boldsymbol{x}_{ \pm}=x_{2}\left(\begin{array}{c}
\cos \kappa \pm\left(B-\sin ^{2} \kappa\right)^{1 / 2} \\
\mathbf{1}
\end{array}\right) \in \mathbb{C}^{B}
$$

with $\mathbf{1}=(1, \ldots, 1)^{T} \in \mathbb{C}^{B-1}$ and $x_{2} \in \mathbb{C} \backslash\{0\}$. It is further calculated in appendix B that

$$
\left\|\boldsymbol{x}_{ \pm}\right\|^{2}=2\left(B-\sin ^{2} \kappa\right)^{1 / 2}\left(\left(B-\sin ^{2} \kappa\right)^{1 / 2} \pm \cos \kappa\right)\left|x_{2}\right|^{2} .
$$

We observe from (3.30) that the absolute value squared of the first component of $\boldsymbol{x}_{ \pm}$ is

$$
\left(\cos \kappa \pm\left(B-\sin ^{2} \kappa\right)^{1 / 2}\right)^{2}\left|x_{2}\right|^{2}=\left(B \pm 2 \sqrt{B} \cos \kappa+\cos 2 \kappa+\mathrm{O}\left(B^{-1 / 2}\right)\right)\left|x_{2}\right|^{2},
$$

and the remaining $B-1$ components have absolute value squared $\left|x_{2}\right|^{2}$.

Using (3.29) and lemma 3.1 we can construct four eigenvectors of $U_{\mathcal{F}}$. We have now collected all the ingredients required to prove our main result for this section:

Proposition 3.3. Consider a star graph with B bonds, a Fourier transform scattering matrix at the central vertex, and incommensurate bond lengths. Define

$$
\begin{aligned}
\boldsymbol{a}^{\mathcal{F}} & :=\boldsymbol{a}^{\mathcal{F}}\left(\kappa, \varepsilon_{1}, \varepsilon_{2}\right) \\
& :=\frac{1}{2 D(\kappa)^{1 / 2}}\left(\begin{array}{c}
\varepsilon_{1} \varepsilon_{2} \mathrm{e}^{\pi \mathrm{i} / 4+\mathrm{i} \kappa / 2+\varepsilon_{2} \mathrm{i} \Phi(\kappa) / 2} N\left(\kappa, \varepsilon_{2}\right)^{1 / 2} \\
\varepsilon_{1} \mathrm{e}^{\pi \mathrm{i} / 4+\mathrm{i} \kappa / 2+\varepsilon_{2} \mathrm{i} \Phi(\kappa) / 2} N\left(\kappa, \varepsilon_{2}\right)^{-1 / 2} \mathbf{1} \\
\varepsilon_{2} \mathrm{e}^{\mathrm{i} \kappa} N\left(\kappa, \varepsilon_{2}\right)^{1 / 2} \\
N\left(\kappa, \varepsilon_{2}\right)^{-1 / 2} \mathbf{1}
\end{array}\right),
\end{aligned}
$$

for any $0<\kappa<\pi / 2, \varepsilon_{1}, \varepsilon_{2} \in\{ \pm 1\}$, where

$$
N\left(\kappa, \varepsilon_{2}\right):=\left(B-\sin ^{2} \kappa\right)^{1 / 2}+\varepsilon_{2} \cos \kappa \quad \text { and } \quad D(\kappa):=\left(B-\sin ^{2} \kappa\right)^{1 / 2},
$$

and $\Phi(\kappa)$ is a function whose value is given by (B.15). Then there is a subsequence $\left(k_{n_{j}}\right) \subseteq\left(k_{n}\right)$ such that the corresponding normalised eigenvectors $\boldsymbol{a}_{n_{j}}$ satisfy

$$
\boldsymbol{a}_{n_{j}} \rightarrow \boldsymbol{a}^{\mathcal{F}}
$$

as $j \rightarrow \infty$. 
Before giving the proof of proposition 3.3, we consider the properties of the limiting eigenvector $\boldsymbol{a}^{\mathcal{F}}$. This will allow us to prove theorem 2.3.

Proof of theorem 2.3. We observe that $\boldsymbol{a}^{\mathcal{F}}$ is a superposition of a vector with equal amplitude across the entire graph, and a component purely localised on the first bond of the graph. From (3.25), the Shannon entropy of $\boldsymbol{a}^{\mathcal{F}}$ is given by

$$
\mathrm{S}\left(\boldsymbol{a}^{\mathcal{F}}\right)=\mathrm{s}\left(\boldsymbol{x}_{ \pm}\right)+\log 2
$$

so we need to calculate $\mathbf{s}\left(\boldsymbol{x}_{ \pm}\right)$.

From the definition (3.22), and (3.31) and (3.32), we can write

$$
\mathbf{s}\left(\boldsymbol{x}_{ \pm}\right)=-\frac{N}{2 D} \log \left(\frac{N}{2 D}\right)-(B-1) \frac{1}{2 N D} \log \left(\frac{1}{2 N D}\right)
$$

where $N$ and $D$ are abbreviations for $N(\kappa, \pm 1)$ and $D(\kappa)$ introduced in (3.34). We write (3.37) in the equivalent form

$$
\mathbf{s}\left(\boldsymbol{x}_{ \pm}\right)=-\frac{1}{2 D}\left(\left(N-\frac{B-1}{N}\right) \log N-\left(N+\frac{B-1}{N}\right) \log 2 D\right)
$$

and given that

$$
\frac{N}{2 D}+\frac{B-1}{2 N D}=1
$$

we have

$$
\mathbf{s}\left(\boldsymbol{x}_{ \pm}\right)=\log 2 D-\frac{N^{2}-(B-1)}{N^{2}+(B-1)} \log N .
$$

With definitions (3.34), (3.40) gives

$$
\mathbf{s}\left(\boldsymbol{x}_{ \pm}\right)=\log 2\left(B-\sin ^{2} \kappa\right)^{1 / 2} \mp \frac{N \cos \kappa}{B-1 \pm N \cos \kappa} \log \left(\left(B-\sin ^{2} \kappa\right)^{1 / 2} \pm \cos \kappa\right),
$$

where we have used

$$
\begin{aligned}
N^{2} & =B-\sin ^{2} \kappa \pm 2 \cos \kappa\left(B-\sin ^{2} \kappa\right)^{1 / 2}+\cos ^{2} \kappa \\
& =B-1 \pm 2\left(B-\sin ^{2} \kappa\right)^{1 / 2} \cos \kappa+2 \cos ^{2} \kappa \\
& =B-1 \pm 2 N \cos \kappa,
\end{aligned}
$$

to simplify. If $B$ is large, then,

$$
\mathrm{s}\left(\boldsymbol{x}_{ \pm}\right)=\frac{1}{2} \log B+\log 2+\mathrm{O}\left(\frac{\log B}{B^{1 / 2}}\right)
$$

This means that for large values of $B$,

$$
\mathrm{S}\left(\boldsymbol{a}^{\mathcal{F}}\right)=\frac{1}{2} \log B+2 \log 2+\mathrm{O}\left(\frac{\log B}{B^{1 / 2}}\right)
$$

essentially (to leading order) meeting the bound (2.13). This proves (2.16). 
By considering the Rényi entropy, we may improve the result further, essentially removing the $\log 2$ term in (3.43). With the same notation as above, for $0 \leqslant \sigma \leqslant 1$,

$$
\begin{aligned}
\mathrm{r}_{\sigma /(1-\sigma)}\left(\boldsymbol{x}_{ \pm}\right)+\mathrm{r}_{-\sigma /(1+\sigma)}\left(\boldsymbol{x}_{ \pm}\right) & \\
= & \frac{\sigma-1}{\sigma} \log \left(\left(\frac{N}{2 D}\right)^{1 /(1-\sigma)}+(B-1)\left(\frac{1}{2 N D}\right)^{1 /(1-\sigma)}\right) \\
& +\frac{\sigma+1}{\sigma} \log \left(\left(\frac{N}{2 D}\right)^{1 /(1+\sigma)}+(B-1)\left(\frac{1}{2 N D}\right)^{1 /(1+\sigma)}\right) \\
= & \frac{\sigma-1}{\sigma} \log \left(N^{1 /(1-\sigma)}+(B-1) N^{-1 /(1-\sigma)}\right) \\
& +\frac{\sigma+1}{\sigma} \log \left(N^{1 /(1+\sigma)}+(B-1) N^{-1 /(1+\sigma)}\right) .
\end{aligned}
$$

Now we specialise to $\sigma \rightarrow 1$. Equation (3.45) yields

$$
r_{\infty}\left(\boldsymbol{x}_{ \pm}\right)+r_{-1 / 2}\left(\boldsymbol{x}_{ \pm}\right)=-\log N+2 \log \left(N^{1 / 2}+(B-1) N^{-1 / 2}\right) .
$$

Inserting $N=\left(\left(B-\sin ^{2} \kappa\right)^{1 / 2} \pm \cos \kappa\right)$ we get

$$
\begin{aligned}
r_{\infty}\left(\boldsymbol{x}_{ \pm}\right)+\mathrm{r}_{-1 / 2}\left(\boldsymbol{x}_{ \pm}\right)= & 2 \log (B-1)-2 \log \left(\left(B-\sin ^{2} \kappa\right)^{1 / 2} \pm \cos \kappa\right) \\
& +2 \log \left(1+\frac{\left(B-\sin ^{2} \kappa\right)^{1 / 2} \pm \cos \kappa}{B-1}\right) \\
= & \log B+\mathrm{O}\left(B^{-1 / 2}\right),
\end{aligned}
$$

for $B$ large. Thus the bound given by (3.26):

$$
\mathbf{r}_{\infty}\left(\boldsymbol{x}_{ \pm}\right)+\mathbf{r}_{-1 / 2}\left(\boldsymbol{x}_{ \pm}\right) \geqslant \log B
$$

is achieved up to terms negligible in the size of the graph. The Rényi entropy of $\boldsymbol{a}^{\mathcal{F}}$ satisfies

$$
\mathrm{R}_{\infty}\left(\boldsymbol{a}^{\mathcal{F}}\right)+\mathrm{R}_{-1 / 2}\left(\boldsymbol{a}^{\mathcal{F}}\right)=\log B+2 \log 2+\mathrm{O}\left(B^{-1 / 2}\right)
$$

as $B \rightarrow \infty$, which is (2.17).

Proof of proposition 3.3. We have

$$
\boldsymbol{a}^{\mathcal{F}}\left(\kappa, \varepsilon_{1}, \varepsilon_{2}\right)=\frac{1}{\sqrt{2}}\left(\begin{array}{c}
\varepsilon_{1} \lambda^{1 / 2} \hat{\boldsymbol{x}}_{ \pm} \\
P(\kappa) \hat{\boldsymbol{x}}_{ \pm}
\end{array}\right)
$$

with $\lambda$ given by (B.16), and the sign \pm chosen according to $\varepsilon_{2}= \pm 1$, and $\hat{\boldsymbol{x}}_{ \pm}$are normalised versions of $\boldsymbol{x}_{ \pm}$from (B.22).

If we choose $k_{0}, L$ such that $k_{0} L_{1}=\kappa$ and $k_{0} L_{j}=1$ for $j=2, \ldots, B$, then $\mathrm{e}^{\mathrm{i} k_{0} L}=P(\kappa)$, and $U_{\mathcal{F}}$ is the quantum evolution operator $D\left(k_{0}, L\right) S$. It follows from lemma 3.1 that $\boldsymbol{a}^{\mathcal{F}}$ is an eigenvector of $U_{\mathcal{F}}$ with eigenvalue $\varepsilon_{1} \lambda^{1 / 2}$. Because $\hat{\boldsymbol{x}}_{ \pm}$are simple eigenvectors, it further follows that $\boldsymbol{a}^{\mathcal{F}}$ is a simple eigenvector of the star graph.

The required convergence then follows from theorem 2.1. 


\subsection{Eigenvectors localised on other bonds}

Proposition 3.3 proves the existence of a limiting eigenvector of a Fourier star graph with enhanced mass concentrated on the first bond of the graph. We can modify the construction to prove the existence of limiting eigenvectors with enhanced mass on a different bond as we now describe.

Let $P_{j}(\kappa)$ be the diagonal $B \times B$ matrix with $j$ th diagonal entry equal to $\mathrm{e}^{\mathrm{i} \kappa}$ and all other entries equal to 1 . This generalises the matrix $P(\kappa)=P_{1}(\kappa)$ introduced in (3.28). The naïve modification of (3.28) with $P_{j}(\kappa)$ instead of $P(\kappa)$ does not produce an eigenvector half-localized on the bond $j$; instead, the result is an eigenvector with half of the mass spread throughout the graph and the other half split equally among the bonds $j$ and $B-j+2$. This is described in more detail in section 4 .

To produce an eigenvector half-localizing on the bond $j$ alone, we will need some extra steps. Let $j \in\{1, \ldots, B\}$ and define the $B \times B$ diagonal matrix

$$
R_{j}=\operatorname{diag}\left\{\mathrm{e}^{-2 \pi \mathrm{i}(j-1)(m-1) / B+\pi \mathrm{i}(j-1)^{2} / B}: m=1, \ldots, B\right\} .
$$

The mnth entry of the matrix $R_{j} \mathcal{F}_{B} R_{j}$ is

$$
\begin{aligned}
& \frac{1}{\sqrt{B}} \mathrm{e}^{-2 \pi \mathrm{i}(j-1)(m-1) / B+\pi \mathrm{i}(j-1)^{2} / B} \mathrm{e}^{2 \pi \mathrm{i}(m-1)(n-1) / B} \mathrm{e}^{-2 \pi \mathrm{i}(j-1)(n-1) / B+\pi \mathrm{i}(j-1)^{2} / B} \\
& =\frac{1}{\sqrt{B}} \mathrm{e}^{2 \pi \mathrm{i}(n-j)(m-j) / B} .
\end{aligned}
$$

In other words, $R_{j} \mathcal{F}_{B} R_{j}$ is a cyclically-permuted version of $\mathcal{F}_{B}$ with the first row and column moved to the $j$ th position. Then we have that $P_{j}(\kappa) R_{j} \mathcal{F}_{B} R_{j} P_{j}(\kappa)$ is a permuted version of $P(\kappa) \mathcal{F}_{B} P(\kappa)$.

By permuting all vectors appearing in the argumentation of section 3.3, we find eigenvectors $\tilde{\boldsymbol{x}}_{ \pm}$with simple eigenvalue $\tilde{\lambda}_{ \pm}$, entry squared (3.32) in the $j$ th component, and $\left|x_{2}\right|^{2}$ in all other components. Because entropy is invariant with respect to permutations, $\mathbf{s}\left(\tilde{\boldsymbol{x}}_{ \pm}\right)=\mathbf{s}\left(\boldsymbol{x}_{ \pm}\right)$as calculated in (3.41), and similarly for $\mathbf{r}_{\rho}\left(\tilde{\boldsymbol{x}}_{ \pm}\right)$.

By lemma 3.1, there are eigenvectors of the matrix

$$
\left(\begin{array}{cc}
0 & P_{j}(\kappa) R_{j} \mathcal{F}_{B} \\
P_{j}(\kappa) R_{j} & 0
\end{array}\right)
$$

of the form

$$
\tilde{\boldsymbol{a}}=\left(\begin{array}{c} 
\pm \tilde{\lambda}_{ \pm}^{1 / 2} \tilde{\boldsymbol{x}}_{ \pm} \\
P_{j}(\kappa) R_{j} \tilde{\boldsymbol{x}}_{ \pm}
\end{array}\right),
$$

and the same entropies as $\boldsymbol{a}^{\mathcal{F}}$, and moreover have enhanced amplitude on the $j$ th bond. These can be shown to be limiting eigenvectors of the Fourier transform star graph by choosing $k_{0}, L_{0}$ such that $D\left(k_{0}, L_{0}\right)=P_{j}(\kappa) R_{j}$ as was done in the proof of proposition 3.3 .

\subsection{Sharpness of the Entropic Uncertainty Principle}

Below the leading order behaviour, there is a difference of $\log 2$ between the Shannon entropy bound arising out of the Entropic Uncertainty Principle and the limiting eigenvectors $\boldsymbol{a}^{\mathcal{F}}$ - compare (2.13) with (2.16). The same is true in fact for the Shannon entropy 


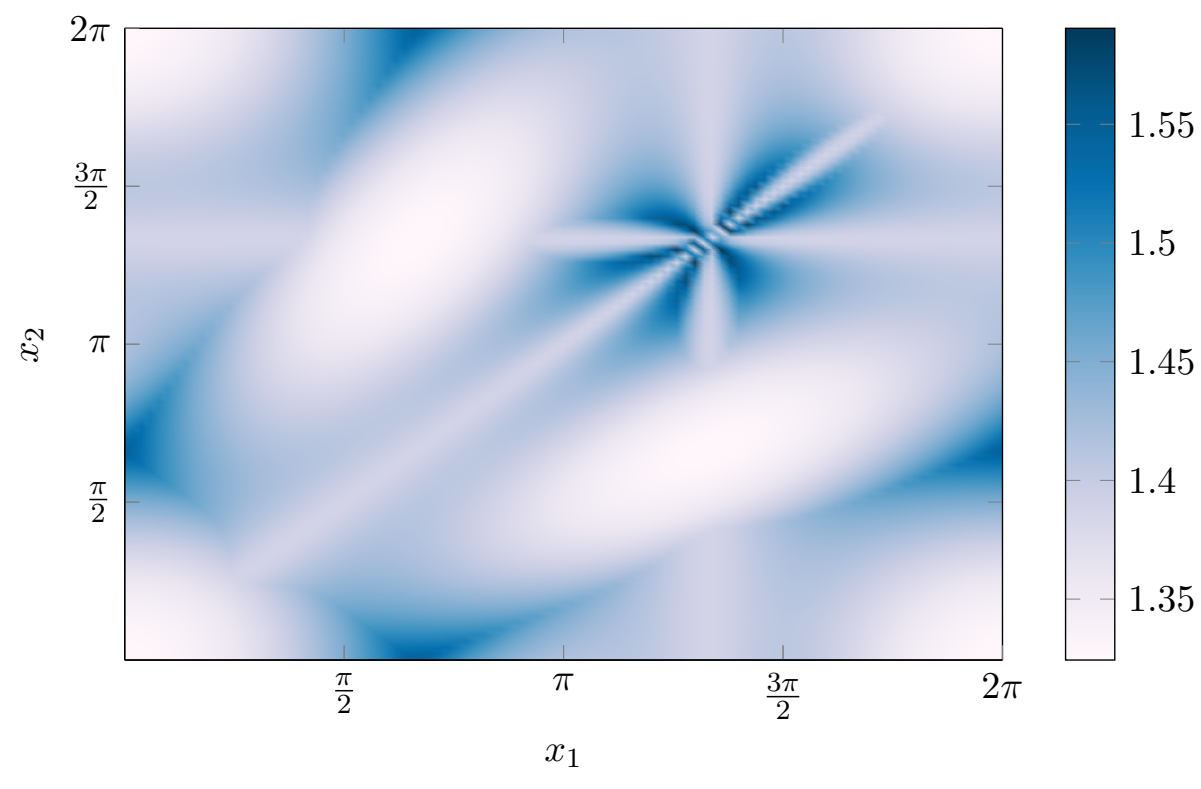

Figure 2: A plot of double the Shannon entropy of the eigenvector with least entropy for the matrices $\Upsilon\left(x_{1}, x_{2}\right) \mathcal{F}_{3}$ where $\Upsilon\left(x_{1}, x_{2}\right)=\operatorname{diag}\left\{1, \mathrm{e}^{\mathrm{i} x_{1}}, \mathrm{e}^{\mathrm{i} x_{2}}\right\}$. Minimal entropy found is approximately $2 \mathrm{~s}\left(\boldsymbol{e}+\boldsymbol{u}_{1}\right) \approx 1.324$ from (3.7). The curious formation at $\left(\frac{4 \pi}{3}, \frac{4 \pi}{3}\right)$ is due to a degeneracy in the spectrum of $\Upsilon \mathcal{F}_{3}$ at this point.

of the limiting eigenvectors on equi-transmitting star graphs (equation (2.19)) and Kirchhoff star graphs (equation (2.21) against the lower-bound (2.22)). This discrepancy could lead to speculation that the Entropic Uncertainty Principle is not sharp. It being prudent to investigate this possibility further we undertook a numerical investigation of the simplest non-trivial case involving the $3 \times 3$ Fourier transform matrix $\mathcal{F}_{3}$. This has the advantages that the matrices are small enough to allow a reasonable exploration of the parameter space, as well as avoiding degeneracies as $\mathcal{F}_{3}$ has a simple spectrum. A basis of eigenvectors of $\mathcal{F}_{3}$ is

$$
\left(\begin{array}{c}
1+\sqrt{3} \\
1 \\
1
\end{array}\right), \quad\left(\begin{array}{c}
1-\sqrt{3} \\
1 \\
1
\end{array}\right), \quad\left(\begin{array}{c}
0 \\
1 \\
-1
\end{array}\right) .
$$

The first listed vector in (3.55) is $\boldsymbol{e}+\boldsymbol{u}_{1}$ as described in section 3.1 and its entropy is (from (3.7))

$$
\mathrm{s}\left(\boldsymbol{e}+\boldsymbol{u}_{1}\right)=\frac{1}{2} \log 3+\log 2-\frac{1}{\sqrt{3}} \log (\sqrt{3}+1) \approx 0.662 .
$$

Our findings may be summarised as follows: the calculations indicate that the the Entropic Uncertainty bound as proved by Maassen and Uffink [47] is sharp in this case; nevertheless our eigenvector results cannot be improved in the sense that there are no Fourier-transform-like matrices with lower entropy eigenvectors.

The sharpness of the first part of Theorem 3.2 when $U=\mathcal{F}_{N}$ is illustrated by taking $\boldsymbol{v}=\boldsymbol{e}_{1}=(1,0,0, \ldots)^{T}$ and, correspondingly, $U \boldsymbol{v}=(1,1,1, \ldots)^{T} / \sqrt{N}$. In this case $\mathrm{R}_{\rho}(\boldsymbol{v})=$ 0 and

$$
\mathrm{R}_{\rho}(U \boldsymbol{v})=-\frac{1}{\rho} \log \left(\sum_{j=1}^{N} N^{-1-\rho}\right)=\log N
$$


independently of $\rho$. The value of the right-hand side of inequalities (3.16)-(3.17) is also $\log N$.

Of course, the vector $\boldsymbol{v}$ chosen above is far from being an eigenvector of $U$ and it is unsurprising that inequalities (3.18)-(3.19) are not sharp. In order to consider if there are Fourier-transform-like matrices with eigenvectors with low entropy we consider the family of matrices $\Upsilon\left(x_{1}, x_{2}\right) \mathcal{F}_{3}$ where

$$
\Upsilon\left(x_{1}, x_{2}\right):=\left(\begin{array}{ccc}
1 & 0 & 0 \\
0 & \mathrm{e}^{\mathrm{i} x_{1}} & 0 \\
0 & 0 & \mathrm{e}^{\mathrm{i} x_{2}}
\end{array}\right) .
$$

This family essentially parameterises the space of $3 \times 3$ unitary matrices with all entries having equal amplitude (such matrices are called complex Hadamard matrices). We plot in figure 2 the Shannon entropy ${ }^{2}$ of the eigenvector with the lowest entropy as $x_{1}, x_{2}$ vary. The minimal entropy we find matches numerically the value $(3.56)$, at $\left(x_{1}, x_{2}\right)=$ $(0,0)$, corresponding to $\mathcal{F}_{3}$ itself, and at $\left(\frac{2 \pi}{3}, \frac{4 \pi}{3}\right)$ and $\left(\frac{4 \pi}{3}, \frac{2 \pi}{3}\right)$ corresponding to cyclic permutations of $\mathcal{F}_{3}$ that were discussed in section 3.4. We conclude that there are not any complex Hadamard matrices with eigenvectors with entropy closer to the bound of $\log 3$ than the Fourier transform matrix and its permutations.

\section{Interpretation and generalisation}

In the previous section we saw that by varying the length of the first bond of the Fourier transform star graph we arrive at quantum limits strongly enhanced by a factor approximately $\sqrt{B}$ on the first bond, with all other components having equal amplitude. To produce localised eigenvectors on other bonds we need to vary all bond lengths as described in section 3.4 .

In this section we explain what happens if we vary the length of a single bond different from the first bond. What we find is an eigenstate that is strongly localised on two bonds of the graph: the $j$ th bond, the one that is varied, and the $(B+2-j)$ th bond. Approximately half the mass is shared equally between those two bonds, and the remaining mass distributed on the remaining bonds.

The analysis turns out to be more complicated, but we are able as a result to give an explanation for the especially simple form (3.30) of perturbed eigenvectors in the case where the first bond length is varied, as well as supplying an alternative proof for why the perturbed eigenvectors are simple. Most of the technical detail is in Appendix C.

Let $P_{j}(\kappa)$ be the diagonal matrix $B \times B$ matrix with $j$ th diagonal entry equal to $\mathrm{e}^{\mathrm{i} \kappa}$ and all other entries equal to 1 as above. Then take

$$
U_{\mathcal{F}}:=U_{\mathcal{F}}(\kappa):=\left(\begin{array}{cc}
0 & P_{j}(\kappa) \mathcal{F}_{B} \\
P_{j}(\kappa) & 0
\end{array}\right)
$$

in place of (3.28). By lemma 3.1, eigenvectors of this $U_{\mathcal{F}}$ are related to the eigenvectors of $P_{j}(\kappa) \mathcal{F}_{B} P_{j}(\kappa)$ through formula (3.10) as we describe below.

Let $F_{j}(\kappa):=P_{j}(\kappa)^{2} \mathcal{F}_{B}$. If $\boldsymbol{f}$ is an eigenvector of $F_{j}(\kappa)$ then $\boldsymbol{x}=P_{j}(\kappa)^{-1} \boldsymbol{f}$ is an eigenvector of $P_{j}(\kappa) \mathcal{F}_{B} P_{j}(\kappa)$ with the same eigenvalue, and moreover

$$
\mathrm{s}(\boldsymbol{x})=\mathrm{s}(\boldsymbol{f}),
$$

\footnotetext{
${ }^{2}$ For computational reasons, the double of the Shannon entropy is actually plotted.
} 
since the entries of $\boldsymbol{x}$ and $\boldsymbol{f}$ are different only by phases.

To an eigenvector $\boldsymbol{f}$ of $F_{j}(\kappa)$ with eigenvalue $\lambda$, lemma 3.1 furnishes eigenvectors

$$
\boldsymbol{a}=\left(\begin{array}{c} 
\pm \lambda^{1 / 2} P_{j}(-\kappa) \boldsymbol{f} \\
\boldsymbol{f}
\end{array}\right)
$$

of $U_{\mathcal{F}}$, and due to (3.25) and (4.2), the Shannon entropy of $\boldsymbol{a}$ is

$$
\mathrm{S}(\boldsymbol{a})=\mathrm{s}(\boldsymbol{f})+\log 2 .
$$

The matrix $F_{j}(\kappa)$ is more beneficial to study because it can be written as a rank-1 perturbation of $\mathcal{F}_{B}$ :

$$
F_{j}(\kappa)=\left(I+\left(\mathrm{e}^{2 \mathrm{i} \kappa}-1\right) \boldsymbol{e}_{j} \boldsymbol{e}_{j}^{\dagger}\right) \mathcal{F}_{B}
$$

with $\boldsymbol{e}_{j}$ as the $j$ th standard $\mathbb{C}^{B}$ basis vector. In particular, the matrix $F_{j}(\kappa)$ coincides with $\mathcal{F}_{B}$ on a subspace of co-dimension 1 - the orthogonal complement to the vector $\boldsymbol{e}_{j}$.

Remark 4.1. Eigenvectors and eigenvalues of rank-1 perturbations can be expressed in terms of those of the unperturbed matrix [52,53] (See also [54]). Usually these calculations are carried out for Hermitian matrices, but the case here with unitary matrices can be done easily analogously, as was done in [55]. The following fact is especially useful to us: if $\mathrm{e}^{\mathrm{i} \phi}$ is an eigenvalue of $\mathcal{F}_{B}$ for which the eigenspace $\operatorname{Eig}\left(\mathcal{F}_{B}, \mathrm{e}^{\mathrm{i} \phi}\right)$ contains a vector $\boldsymbol{u}$ with $\boldsymbol{e}_{j}^{\dagger} \boldsymbol{u}=0$, then $\mathrm{e}^{\mathrm{i} \phi}$ is an eigenvalue of $F_{j}(\kappa)$ with multiplicity $\operatorname{dim} \operatorname{Eig}\left(\mathcal{F}_{B}, \mathrm{e}^{\mathrm{i} \phi}\right)-1$. We remark that this is valid for all $\kappa$ and also valid in the case when $\operatorname{dim} \operatorname{Eig}\left(\mathcal{F}_{B}, \mathrm{e}^{\mathrm{i} \phi}\right)=1$, in which case $\mathrm{e}^{\mathrm{i} \phi}$ is not an eigenvalue of $F_{j}(\kappa)$.

It follows that we need to understand the eigenvalues of the Fourier matrix $\mathcal{F}_{B}$ (see [50] for more complete information). Explicit computation shows that $\mathcal{F}_{B}^{2}$ is the matrix of the permutation $\left(\begin{array}{ccccccc}1 & 2 & 3 & \ldots & B-1 & B \\ 1 & B & B-1 & \ldots & 3 & 2\end{array}\right)$. This permutation leaves invariant the element 1 and, if $B$ is even, the element $B / 2+1$. Since it is an involution, we conclude that $\mathcal{F}_{B}^{4}=I$ and the spectrum of $\mathcal{F}_{B}$ consists of the numbers \pm 1 and $\pm \mathrm{i}$.

We start with the case $j=1$. Let $\boldsymbol{e}=(1,1,1, \ldots)^{T}$ and $\boldsymbol{u}_{1}=\boldsymbol{e}_{1}$. As noted in (3.6), the vectors $\boldsymbol{e} \pm \boldsymbol{u}_{1}$ are eigenvectors of $\mathcal{F}_{B}$ with eigenvalues \pm 1 . Complete these two vectors to an orthonormal basis $\left\{\boldsymbol{e}+\boldsymbol{u}_{1}, \boldsymbol{e}-\boldsymbol{u}_{1}, \boldsymbol{w}_{3}, \ldots, \boldsymbol{w}_{B}\right\}$ of $\mathcal{F}_{B}$, which we can always do because $\mathcal{F}_{B}$ is unitary. Since $\boldsymbol{e}_{1}=\boldsymbol{u}_{1}$ is a linear combination of the first two vectors, each of the eigenvectors $\boldsymbol{w}_{3}, \ldots, \boldsymbol{w}_{B}$ is orthogonal to $\boldsymbol{e}_{1}$ and therefore still an eigenvector of $F_{1}(\kappa)$ with the same eigenvalue. The two other eigenvalues of $F_{1}(\kappa)$ must be different from \pm 1 by Remark 4.1 but must remain close to \pm 1 for small $\kappa$ (by classical perturbation theory results). This is shown schematically in figure $3(\mathrm{a})$. The corresponding eigenvectors must remain orthogonal to $\boldsymbol{w}_{3}, \ldots, \boldsymbol{w}_{B}$ and are therefore linear combinations of $\boldsymbol{e} \pm \boldsymbol{u}_{1}$, i.e. can be written in the general form,

$$
\boldsymbol{x}=\left(\begin{array}{c}
x_{1} \\
x_{2} \mathbf{1}
\end{array}\right), \quad x_{1}, x_{2} \in \mathbb{C} .
$$

This explains the form of the eigenvectors in equation (3.30). We get qualitatively similar results as in section 3.3 if $B$ is even and $j=B / 2+1$.

Now take general $j$ different from 1 and (if $B$ is even) from $B / 2+1$. Suppose for simplicity that $B \geqslant 5$. Let $\boldsymbol{u}_{j} \in \mathbb{C}^{B}$ be the vector with 1 s in the $j$ th and $(B+2-j)$ th entries, and zeros in all other positions, and $\boldsymbol{v}_{j} \in \mathbb{C}^{B}$ be the vector with a 1 in $j$ th position, 


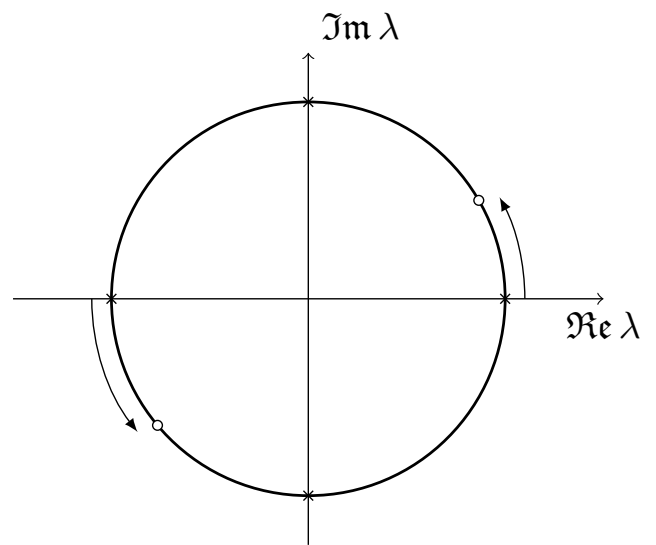

(a)

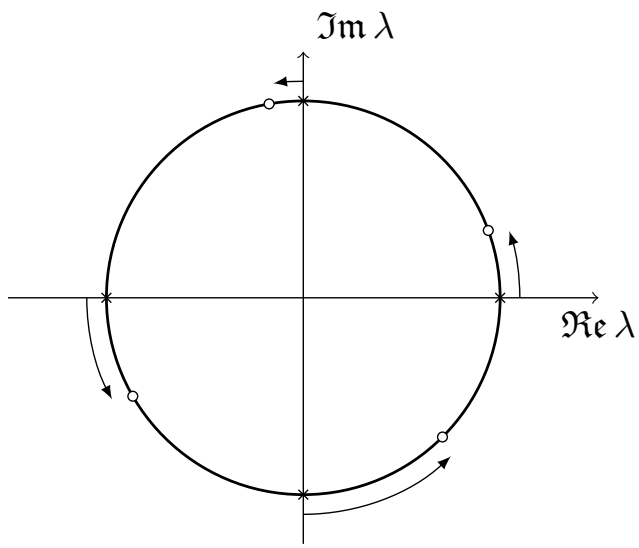

(b)

Figure 3: Schematic depiction of eigenvalues of the matrix $F_{j}(\kappa)$ as a function of $\kappa$ when $j=1$ and when $j \neq 1$.

-1 in the $(B+2-j)$ th position, and zeros in all other entries. For example, in the case $j=2$ we have

$$
\boldsymbol{u}_{2}=\left(\begin{array}{c}
0 \\
1 \\
0 \\
\vdots \\
0 \\
1
\end{array}\right) \quad \text { and } \quad \boldsymbol{v}_{2}=\left(\begin{array}{c}
0 \\
1 \\
0 \\
\vdots \\
0 \\
-1
\end{array}\right)
$$

Then define

$$
\begin{aligned}
\boldsymbol{w}_{+} & :=\mathcal{F}_{B} \boldsymbol{u}_{j}+\boldsymbol{u}_{j} \\
\boldsymbol{w}_{-} & :=\mathcal{F}_{B} \boldsymbol{u}_{j}-\boldsymbol{u}_{j} \\
\boldsymbol{w}_{+\mathrm{i}} & :=\mathrm{i} \mathcal{F}_{B} \boldsymbol{v}_{j}-\boldsymbol{v}_{j} \\
\boldsymbol{w}_{-\mathrm{i}} & :=\mathrm{i} \mathcal{F}_{B} \boldsymbol{v}_{j}+\boldsymbol{v}_{j} .
\end{aligned}
$$

If $B \geqslant 5$, these vectors are non-zero. Noting that $\mathcal{F}_{B}^{2} \boldsymbol{u}_{j}=\boldsymbol{u}_{j}$ and $\mathcal{F}_{B}^{2} \boldsymbol{v}_{j}=-\boldsymbol{v}_{j}$ (by the permutation properties of $\mathcal{F}_{B}^{2}$ ), we see that $\boldsymbol{w}_{+}, \boldsymbol{w}_{-}, \boldsymbol{w}_{+\mathrm{i}}, \boldsymbol{w}_{-\mathrm{i}}$ are eigenvectors of $\mathcal{F}_{B}$ with eigenvalues respectively: $+1,-1,+\mathrm{i},-\mathrm{i}$. Proceed as before: complete this set to find an orthonormal basis

$$
\left\{\boldsymbol{w}_{+}, \boldsymbol{w}_{-}, \boldsymbol{w}_{+\mathrm{i}}, \boldsymbol{w}_{-\mathrm{i}}, \boldsymbol{w}_{5}, \ldots \boldsymbol{w}_{B}\right\}
$$

Since

$$
\boldsymbol{e}_{j}=\frac{1}{4}\left(\boldsymbol{w}_{+}-\boldsymbol{w}_{-}-\boldsymbol{w}_{+\mathrm{i}}+\boldsymbol{w}_{-\mathrm{i}}\right)
$$

the eigenvectors $\boldsymbol{w}_{5}, \ldots \boldsymbol{w}_{B}$ are orthogonal to $\boldsymbol{e}_{j}$ and thus remain eigenvectors of the perturbed matrix $F_{j}(\kappa)$. The other eigenvalues of $F_{j}(\kappa)$ must be different from \pm 1 and $\pm \mathrm{i}$ by Remark 4.1. In fact, they move with $\kappa$ as indicated in figure 3(b). The corresponding eigenvectors are linear combinations of $\left\{\boldsymbol{w}_{+}, \boldsymbol{w}_{-}, \boldsymbol{w}_{+\mathrm{i}}, \boldsymbol{w}_{-\mathrm{i}}\right\}$, and therefore can be written in the form

$$
\boldsymbol{f}=\beta_{1} \mathcal{F}_{B} \boldsymbol{u}_{j}+\beta_{2} \mathrm{i} \mathcal{F}_{B} \boldsymbol{v}_{j}+\beta_{3} \boldsymbol{u}_{j}+\beta_{4} \boldsymbol{v}_{j},
$$


A calculation presented in appendix $\mathrm{C}$ results in

$$
\begin{aligned}
& \beta_{1}=Z \mathrm{e}^{-\mathrm{i} \phi} \cot \phi \\
& \beta_{2}=Z \mathrm{e}^{-\mathrm{i} \phi} \\
& \beta_{3}=Z \cot \phi \\
& \beta_{4}=\mathrm{i} Z,
\end{aligned}
$$

where $Z \in \mathbb{C} \backslash\{0\}$, and $\mathrm{e}^{\mathrm{i} \phi}$ is the perturbed eigenvalue, which is one of the solutions $\lambda$ to

$$
\lambda^{4}-\frac{\lambda^{3}\left(\mathrm{e}^{2 \mathrm{i} \kappa}-1\right)}{\sqrt{B}} \mathrm{e}^{2 \pi \mathrm{i}(j-1)^{2} / B}-\frac{\lambda\left(\mathrm{e}^{2 \mathrm{i} \kappa}-1\right)}{\sqrt{B}} \mathrm{e}^{-2 \pi \mathrm{i}(j-1)^{2} / B}-\mathrm{e}^{2 \mathrm{i} \kappa}=0 .
$$

The norm of $\boldsymbol{f}$ is

$$
\|\boldsymbol{f}\|^{2}=|Z|^{2} \operatorname{cosec}^{2} \phi\left(4+\frac{8}{\sqrt{B}}\left(\cos ^{3} \phi \cos \left(\frac{2 \pi(j-1)^{2}}{B}\right)+\sin ^{3} \phi \sin \left(\frac{2 \pi(j-1)^{2}}{B}\right)\right)\right)
$$

and the entropy satisfies

$$
\mathbf{s}(\boldsymbol{f})=\frac{1}{2} \log B+2 \log 2-\frac{1}{2}+\mathrm{O}\left(\frac{\log B}{B^{1 / 2}}\right) .
$$

The corresponding entropy of the vector $\boldsymbol{a}$ given by (4.3) is, from (4.4),

$$
\mathrm{S}(\boldsymbol{a})=\frac{1}{2} \log B+3 \log 2-\frac{1}{2}+\mathrm{O}\left(\frac{\log B}{B^{1 / 2}}\right) .
$$

We observe that this is further from the theoretical bound (2.13) than we could achieve by varying the first bond, as reported in section 3.3. The perturbed eigenfunctions now have approximately half the mass concentrated on the $j$ th and the $(B+2-j)$ th bond, and the remaining components are not equal, but vary from bond-to-bond. This is the explanation for why the entropy (4.16) is further from the bound; the difference is a fundamental consequence of the shape of the eigenfunctions and cannot be removed by switching to a different entropy.

\section{Equi-transmitting boundary conditions}

We now turn our attention to quantum star graphs with an equi-transmitting scattering matrix at the centre. To that effect we consider the $2 B \times 2 B$ unitary matrices of the form

$$
U_{E}:=U_{E}(\kappa):=\left(\begin{array}{cc}
0 & P(\kappa) E_{B} \\
P(\kappa) & 0
\end{array}\right)
$$

where $E_{B}$ is an equi-transmitting matrix of the form (3.3).

Proposition 5.1. Consider a star graph with $B$ bonds, an equi-transmitting scattering matrix $E_{B}$ of the form (3.3) at the central vertex, and incommensurate bond lengths. Let

$$
\boldsymbol{a}^{E}:=\boldsymbol{a}^{E}\left(\kappa, \varepsilon_{1}, \varepsilon_{2}\right):=\frac{1}{2 \sqrt{B-1}}\left(\begin{array}{c}
\varepsilon_{1} \varepsilon_{2} \mathrm{e}^{\pi \mathrm{i}\left(1-\varepsilon_{2}\right) / 4+\mathrm{i} \kappa / 2} \sqrt{B-1} \\
\varepsilon_{1} \mathrm{e}^{\pi \mathrm{i}\left(1-\varepsilon_{2}\right) / 4+\mathrm{i} \kappa / 2} \mathbf{1} \\
\varepsilon_{2} \mathrm{e}^{\mathrm{i} \kappa} \sqrt{B-1} \\
\mathbf{1}
\end{array}\right)
$$


for any $\varepsilon_{1}, \varepsilon_{2} \in\{ \pm 1\}$ and $0<\kappa \leqslant \pi$ such that $\varepsilon_{2} \mathrm{e}^{\mathrm{i} \kappa}$ is not an eigenvalue of $E_{B}$. Then there is a subsequence $\left(k_{n_{j}}\right) \subseteq\left(k_{n}\right)$ such that the corresponding normalised eigenvectors $\boldsymbol{a}_{n_{j}}$ satisfy

$$
\boldsymbol{a}_{n_{j}} \rightarrow \boldsymbol{a}^{E}
$$

as $j \rightarrow \infty$.

Proof. Eigenvectors of $U_{E}$ are, by lemma 3.1, of the form

$$
\boldsymbol{a}=\left(\begin{array}{c} 
\pm \mu^{1 / 2} \boldsymbol{y} \\
P(\kappa) \boldsymbol{y}
\end{array}\right)
$$

where $\boldsymbol{y}$ is an eigenvector of $P(\kappa) E_{B} P(\kappa)$ with eigenvalue $\mu \in \mathbb{T}$.

If we suppose

$$
\boldsymbol{y}=\left(\begin{array}{c}
y_{1} \\
y_{2} \mathbf{1}
\end{array}\right)=y_{1} \boldsymbol{u}_{1}+\sqrt{B-1} y_{2} \tilde{\boldsymbol{u}}_{1}
$$

using definitions (3.4). We have

$$
P(\kappa) \boldsymbol{y}=\left(\begin{array}{c}
\mathrm{e}^{\mathrm{i} \kappa} y_{1} \\
y_{2} \mathbf{1}
\end{array}\right)=\mathrm{e}^{\mathrm{i} \kappa} y_{1} \boldsymbol{u}_{1}+\sqrt{B-1} y_{2} \tilde{\boldsymbol{u}}_{1}
$$

and by (3.8),

$$
\begin{aligned}
E_{B} P(\kappa) \boldsymbol{y} & =\mathrm{e}^{\mathrm{i} \kappa} y_{1} \tilde{\boldsymbol{u}}_{1}+\sqrt{B-1} y_{2} \boldsymbol{u}_{1} \\
& =\left(\begin{array}{c}
y_{2} \sqrt{B-1} \\
\frac{1}{\sqrt{B-1}} \mathrm{e}^{\mathrm{i} \kappa} y_{1} \mathbf{1}
\end{array}\right) .
\end{aligned}
$$

Finally,

$$
P(\kappa) E_{B} P(\kappa) \boldsymbol{y}=\left(\begin{array}{c}
\mathrm{e}^{\mathrm{i} \kappa} y_{2} \sqrt{B-1} \\
\frac{1}{\sqrt{B-1}} \mathrm{e}^{\mathrm{i} \kappa} y_{1} \mathbf{1}
\end{array}\right) .
$$

The eigenequation $P(\kappa) E_{B} P(\kappa) \boldsymbol{y}=\mu \boldsymbol{y}$ is soluble in non-zero $\boldsymbol{y}$ if and only if $\mu$ satisfies

$$
\begin{aligned}
& \mathrm{e}^{\mathrm{i} \kappa} y_{2} \sqrt{B-1}=\mu y_{1}, \\
& \frac{1}{\sqrt{B-1}} \mathrm{e}^{\mathrm{i} \kappa} y_{1}=\mu y_{2} .
\end{aligned}
$$

The system (5.9) requires $\mu^{2}=\mathrm{e}^{2 \mathrm{i} \kappa}$, whence

$$
\mu= \pm \mathrm{e}^{\mathrm{i} \kappa}
$$

and

$$
y_{1}= \pm \sqrt{B-1} y_{2}
$$

Define

$$
\boldsymbol{y}_{ \pm}:=y_{2}\left(\begin{array}{c} 
\pm \sqrt{B-1} \\
\mathbf{1}
\end{array}\right)
$$

and the normalised versions

$$
\hat{\boldsymbol{y}}_{ \pm}:=\frac{1}{\sqrt{2(B-1)}}\left(\begin{array}{c} 
\pm \sqrt{B-1} \\
\mathbf{1}
\end{array}\right) .
$$


The vectors $\boldsymbol{y}_{ \pm}$(and $\hat{\boldsymbol{y}}_{ \pm}$) are eigenvectors with eigenvalue $\pm \mathrm{e}^{\mathrm{i} \kappa}$. Moreover, it follows from the same argument as in section 4 that all other eigenvectors of $P(\kappa) E_{B} P(\kappa)$ are eigenvectors of $E_{B}$, so, provided that $\pm \mathrm{e}^{\mathrm{i} \kappa}$ is not an eigenvalue of $E_{B}, \boldsymbol{y}_{ \pm}$is simple.

The vector $\boldsymbol{a}^{E}$ is given by

$$
\boldsymbol{a}^{E}=\frac{1}{\sqrt{2}}\left(\begin{array}{c}
\varepsilon_{1} \mu^{1 / 2} \hat{\boldsymbol{y}}_{ \pm} \\
P(\kappa) \hat{\boldsymbol{y}}_{ \pm}
\end{array}\right)
$$

with the \pm sign chosen according to $\varepsilon_{2}= \pm 1$. Due to the above it may be deduced that this is a simple eigenvector of $U_{E}$. For choice of bond lengths and $k_{0}$ as in the proof of proposition $3.3, \boldsymbol{a}^{E}$ is a simple eigenvector of the quantum evolution operator, and hence we get the convergence (5.3) due to theorem 2.1.

For certain values ${ }^{3}$ of $B$ it is possible to find equi-transmitting matrices $E_{B}$ that are symmetric. For such matrices, \pm 1 are the only eigenvalues and hence any $\kappa$ with $0<\kappa<\pi$ can be taken in proposition 5.1. The matrix $E_{6}$ shown in (1.1) is such an example.

Proof of theorem 2.4. From (3.25) and (5.14),

$$
\mathrm{S}\left(\boldsymbol{a}^{E}\right)=\mathbf{s}\left(\hat{\boldsymbol{y}}_{ \pm}\right)+\log 2
$$

where $\mathbf{s}\left(\hat{\boldsymbol{y}}_{ \pm}\right)$may be calculated from (3.40) except that now

$$
N:=D:=\sqrt{B-1} .
$$

We get

$$
\mathbf{s}\left(\hat{\boldsymbol{y}}_{ \pm}\right)=\frac{1}{2} \log (B-1)+\log 2 .
$$

This leads to

$$
\mathrm{S}\left(\boldsymbol{a}^{E}\right)=\frac{1}{2} \log (B-1)+2 \log 2,
$$

which is (2.19) and should be compared with the theoretical bound (2.14) for equitransmitting star graph eigenvectors. Again, to leading order, the bound has been reached. However, we can again improve this by considering the Rényi entropy. If we consider (3.46) with $N, D$ as in (5.16) for the case $\hat{\boldsymbol{y}}_{ \pm}$, we obtain

$$
\begin{aligned}
\mathbf{r}_{\infty}\left(\hat{\boldsymbol{y}}_{ \pm}\right)+\mathrm{r}_{-1 / 2}\left(\hat{\boldsymbol{y}}_{ \pm}\right) & =-\frac{1}{2} \log (B-1)+2 \log \left((B-1)^{1 / 4}+(B-1)^{3 / 4}\right) \\
& =\log (B-1)+2 \log \left(1+(B-1)^{-1 / 2}\right)
\end{aligned}
$$

for large values of $B$. We have achieved the bound (3.27) up to terms negligible in $B$. For the Rényi entropy of $\boldsymbol{a}^{E}$ we have

$$
\mathrm{R}_{\infty}\left(\boldsymbol{a}^{E}\right)+\mathrm{R}_{-1 / 2}\left(\boldsymbol{a}^{E}\right)=\log (B-1)+2 \log 2+2 \log \left(1+(B-1)^{-1 / 2}\right) .
$$

which leads to (2.20).

These results mirror the results that we proved in section 3.3 for the Fourier transform scattering matrix, and varying the length of the first bond. Because we do not have complete information about the spectrum and eigenvectors of general equi-transmitting matrices, we cannot repeat the analysis of the vectors reached by varying other bond lengths than the first one. Whether it is possible to do this in specific cases, such as when the equi-transmitting matrices are constructed from Dirichlet characters [35] where $B-1$ is a prime number is an interesting question that we leave for future study.

\footnotetext{
${ }^{3}$ Specifically if $B \equiv 2 \bmod 4$ with $B-1$ a prime; see Corollary 3.5 of [35].
} 


\section{Acknowledgements}

The authors are grateful to Stéphane Nonnenmacher for a helpful discussion of the Entropic Uncertainty Principle. GB acknowledges partial support from the NSF under grant DMS1410657.

\section{A Bond-scattering matrix of a star graph}

We suppose a star graph with $B$ bonds (and $B+1$ vertices) has at the central vertex the scattering matrix $\Sigma$, and we suppose Kirchhoff conditions at the leaves (exterior vertices). For vertices of degree one, this condition reduces to simple reflection of waves. The argument below can be modified easily to allow other conditions at the leaves.

We order the directed bonds so that the first $B$ bonds point inwards to the central vertex, and the subsequent bonds are the reversals. The wave-function for $k>0$ on the $j$ th bond can be written

$$
\psi_{j}(x)=a_{j}^{\text {in }} \mathrm{e}^{-\mathrm{i} k x}+a_{j}^{\text {out }} \mathrm{e}^{\mathrm{i} k x} .
$$

and because we will be assuming bond $B+j$ is the reversal of bond $j$,

$$
\begin{aligned}
\psi_{B+j}(x) & =\psi_{j}\left(L_{j}-x\right) \\
& =a_{j}^{\text {in }} \mathrm{e}^{-\mathrm{i} k L_{j}+\mathrm{i} k x}+a_{j}^{\text {out }} \mathrm{e}^{\mathrm{i} k L_{j}-\mathrm{i} k x},
\end{aligned}
$$

whence, for $1 \leqslant j \leqslant B$,

$$
a_{j+B}^{\text {in }}=\mathrm{e}^{\mathrm{i} k L_{j}} a_{j}^{\text {out }} \quad \text { and } \quad a_{j+B}^{\text {out }}=\mathrm{e}^{-\mathrm{i} k L_{j}} a_{j}^{\text {in }} .
$$

The $B \times B$ matrix $\Sigma$ controls scattering at the central vertex so

$$
\begin{aligned}
\Sigma\left(\begin{array}{c}
a_{B+1}^{\text {in }} \\
\vdots \\
a_{2 B}^{\text {in }}
\end{array}\right) & =\left(\begin{array}{c}
a_{B+1}^{\text {out }} \\
\vdots \\
a_{2 B}^{\text {out }}
\end{array}\right) \\
& =\left(\begin{array}{c}
\mathrm{e}^{-\mathrm{i} k L_{1}} a_{1}^{\text {in }} \\
\vdots \\
\mathrm{e}^{-\mathrm{i} k L_{B}} a_{B}^{\text {in }}
\end{array}\right) \quad \text { using }(\mathrm{A} .3), \\
& =\operatorname{diag}\left\{\mathrm{e}^{-\mathrm{i} k L_{1}}, \ldots, \mathrm{e}^{-\mathrm{i} k L_{B}}\right\}\left(\begin{array}{c}
a_{1}^{\text {in }} \\
\vdots \\
a_{B}^{\text {in }}
\end{array}\right) .
\end{aligned}
$$

At the leaves of the star graph waves are reflected which imposes

$$
\begin{aligned}
a_{j}^{\text {in }} & =a_{j}^{\text {out }}, \quad j=1, \ldots, B, \\
& =\mathrm{e}^{-\mathrm{i} k L_{j}} a_{j+B}^{\text {in }},
\end{aligned}
$$

using (A.3) once more. We have

$$
\left(\begin{array}{c}
a_{1}^{\mathrm{in}} \\
\vdots \\
a_{B}^{\mathrm{in}}
\end{array}\right)=\left(\begin{array}{c}
\mathrm{e}^{-\mathrm{i} k L_{1}} a_{B+1}^{\mathrm{in}} \\
\vdots \\
\mathrm{e}^{-\mathrm{i} k L_{B}} a_{2 B}^{\mathrm{in}}
\end{array}\right)=\operatorname{diag}\left\{\mathrm{e}^{-\mathrm{i} k L_{1}}, \ldots, \mathrm{e}^{-\mathrm{i} k L_{B}}\right\}\left(\begin{array}{c}
a_{B+1}^{\mathrm{in}} \\
\vdots \\
a_{2 B}^{\mathrm{in}}
\end{array}\right) .
$$


We can combine (A.4) and (A.6) into a single system: denoting

$$
\boldsymbol{a}=\left(a_{1}^{\mathrm{in}}, a_{2}^{\mathrm{in}}, \ldots, a_{2 B}^{\mathrm{in}}\right)^{T},
$$

we have

$$
D(-k) \boldsymbol{a}=\left(\begin{array}{cc}
0 & \Sigma \\
I & 0
\end{array}\right) \boldsymbol{a} .
$$

Equivalently, $(I-D(k) S) \boldsymbol{a}=0$, with

$$
S=\left(\begin{array}{cc}
0 & \Sigma \\
I & 0
\end{array}\right)
$$

the bond scattering matrix.

\section{B Perturbing the length of the first bond}

In this appendix we calculate eigenvectors $\boldsymbol{x}$ of the matrix $P(\kappa) \mathcal{F}_{B} P(\kappa)$ introduced in section 3.3. Let us look for $\boldsymbol{x}$ of the form

$$
\boldsymbol{x}=\left(\begin{array}{c}
x_{1} \\
x_{2} \mathbf{1}
\end{array}\right) \in \mathbb{C}^{B}
$$

where $x_{1}, x_{2} \in \mathbb{C}$ and $\mathbf{1}=(1, \ldots, 1)^{T} \in \mathbb{C}^{B-1}$. We have

$$
P(\kappa) \boldsymbol{x}=\left(\begin{array}{c}
\mathrm{e}^{\mathrm{i} \kappa} x_{1} \\
x_{2} \mathbf{1}
\end{array}\right)
$$

We can write

$$
\left(\begin{array}{c}
\mathrm{e}^{\mathrm{i} \kappa} x_{1} \\
\mathbf{1} x_{2}
\end{array}\right)=\sqrt{B} x_{2} \boldsymbol{e}+\left(\mathrm{e}^{\mathrm{i} \kappa} x_{1}-x_{2}\right) \boldsymbol{u}_{1}
$$

so that it is easy to see from (3.5) that

$$
\mathcal{F}_{B} P(\kappa) \boldsymbol{x}=\sqrt{B} x_{2} \boldsymbol{u}_{1}+\left(\mathrm{e}^{\mathrm{i} \kappa} x_{1}-x_{2}\right) \boldsymbol{e}
$$

Thus, the first component of $P(\kappa) \mathcal{F}_{B} P(\kappa) \boldsymbol{x}$ is

$$
\mathrm{e}^{\mathrm{i} \kappa}\left(\sqrt{B} x_{2}+\frac{\mathrm{e}^{\mathrm{i} \kappa} x_{1}-x_{2}}{\sqrt{B}}\right)
$$

and all remaining components take the same value $\left(\mathrm{e}^{\mathrm{i} \kappa} x_{1}-x_{2}\right) / \sqrt{B}$. The eigenvector equation

$$
P(\kappa) \mathcal{F}_{B} P(\kappa) \boldsymbol{x}=\lambda \boldsymbol{x},
$$

can be expressed using (B.1) as the $2 \times 2$ linear system

$$
\left(\begin{array}{cc}
\mathrm{e}^{2 \mathrm{i} \kappa} / \sqrt{B} & \mathrm{e}^{\mathrm{i} \kappa}(B-1) / \sqrt{B} \\
\mathrm{e}^{\mathrm{i} \kappa} / \sqrt{B} & -1 / \sqrt{B}
\end{array}\right)\left(\begin{array}{l}
x_{1} \\
x_{2}
\end{array}\right)=\lambda\left(\begin{array}{l}
x_{1} \\
x_{2}
\end{array}\right) .
$$

The characteristic equation of the matrix in (B.7) is

$$
\operatorname{det}\left(\begin{array}{cc}
\lambda-\frac{\mathrm{e}^{2 \mathrm{i} \kappa}}{\sqrt{B}} & -\mathrm{e}^{\mathrm{i} \kappa} \frac{B-1}{\sqrt{B}} \\
-\frac{\mathrm{e}^{\mathrm{i} \kappa}}{\sqrt{B}} & \lambda+\frac{1}{\sqrt{B}}
\end{array}\right)=0
$$


which can be expressed as

$$
\begin{array}{r}
\left(\lambda-\frac{\mathrm{e}^{2 \mathrm{i} \kappa}}{\sqrt{B}}\right)\left(\lambda+\frac{1}{\sqrt{B}}\right)-\mathrm{e}^{2 \mathrm{i} \kappa} \frac{B-1}{B}=0 \\
\Rightarrow \quad \lambda^{2}-\frac{1}{\sqrt{B}}\left(\mathrm{e}^{2 \mathrm{i} \kappa}-1\right) \lambda-\mathrm{e}^{2 \mathrm{i} \kappa}=0 .
\end{array}
$$

Now

$$
\begin{aligned}
\mathrm{e}^{2 \mathrm{i} \kappa}-1 & =\mathrm{e}^{\mathrm{i} \kappa}\left(\mathrm{e}^{\mathrm{i} \kappa}-\mathrm{e}^{-\mathrm{i} \kappa}\right) \\
& =2 \mathrm{ie}^{\mathrm{i} \kappa} \sin \kappa,
\end{aligned}
$$

so we have

$$
\left(\mathrm{e}^{2 \mathrm{i} \kappa}-1\right)^{2}=-4 \sin ^{2} \kappa \mathrm{e}^{2 \mathrm{i} \kappa},
$$

and (for substitution into the quadratic formula)

$$
\frac{1}{B}\left(\mathrm{e}^{2 \mathrm{i} \kappa}-1\right)^{2}+4 \mathrm{e}^{2 \mathrm{i} \kappa}=4\left(1-\frac{1}{B} \sin ^{2} \kappa\right) \mathrm{e}^{2 \mathrm{i} \kappa} .
$$

The solutions $\lambda$ to (B.9) satisfy

$$
\begin{aligned}
\lambda & =\frac{B^{-1 / 2}\left(\mathrm{e}^{2 \mathrm{i} \kappa}-1\right) \pm 2\left(1-B^{-1} \sin ^{2} \kappa\right)^{1 / 2} \mathrm{e}^{\mathrm{i} \kappa}}{2} \\
& =\frac{\mathrm{e}^{2 \mathrm{i} \kappa}-1 \pm 2\left(B-\sin ^{2} \kappa\right)^{1 / 2} \mathrm{e}^{\mathrm{i} \kappa}}{2 \sqrt{B}} \\
& =\frac{2\left( \pm\left(B-\sin ^{2} \kappa\right)^{1 / 2}+\mathrm{i} \sin \kappa\right) \mathrm{e}^{\mathrm{i} \kappa}}{2 \sqrt{B}},
\end{aligned}
$$

using (B.10). Writing

$$
\left( \pm\left(B-\sin ^{2} \kappa\right)^{1 / 2}+\mathrm{i} \sin \kappa\right)=\sqrt{B} \mathrm{e}^{\pi \mathrm{i} / 2 \pm \mathrm{i} \Phi(\kappa)}
$$

where

$$
\tan \Phi(\kappa):=-\frac{\sqrt{B-\sin ^{2} \kappa}}{\sin \kappa},
$$

we get

$$
\lambda=\mathrm{e}^{\pi \mathrm{i} / 2+\mathrm{i} \kappa \pm \mathrm{i} \Phi(\kappa)}
$$

and neither of the two values of $\lambda$ are in the set $\{ \pm 1, \pm \mathrm{i}\}$, if $0<\kappa<\pi / 2$.

Let us now turn to the eigenvectors corresponding to the eigenvalues $\lambda$. From (B.7), we get

$$
\begin{aligned}
\mathrm{e}^{\mathrm{i} \kappa} B x_{2}+\mathrm{e}^{2 \mathrm{i} \kappa} x_{1}-\mathrm{e}^{\mathrm{i} \kappa} x_{2} & =\lambda \sqrt{B} x_{1} \\
\mathrm{e}^{\mathrm{i} \kappa} x_{1}-x_{2} & =\lambda \sqrt{B} x_{2},
\end{aligned}
$$

which are equivalent to

$$
x_{1}=\mathrm{e}^{-\mathrm{i} \kappa}(\lambda \sqrt{B}+1) x_{2} .
$$

We note that from (B.13),

$$
\begin{aligned}
\lambda \sqrt{B}+1 & =\left( \pm\left(B-\sin ^{2} \kappa\right)^{1 / 2}+\mathrm{i} \sin \kappa+\mathrm{e}^{-\mathrm{i} \kappa}\right) \mathrm{e}^{\mathrm{i} \kappa} \\
& =\left(\cos \kappa \pm\left(B-\sin ^{2} \kappa\right)^{1 / 2}\right) \mathrm{e}^{\mathrm{i} \kappa},
\end{aligned}
$$


so (B.18) becomes

$$
x_{1}=\left( \pm\left(B-\sin ^{2} \kappa\right)^{1 / 2}+\cos \kappa\right) x_{2} .
$$

The upshot is that we find eigenvectors of $P(\kappa) \mathcal{F}_{B} P(\kappa)$ of the form

$$
\boldsymbol{x}_{ \pm}=x_{2}\left(\begin{array}{c}
\cos \kappa \pm\left(B-\sin ^{2} \kappa\right)^{1 / 2} \\
\mathbf{1}
\end{array}\right) \in \mathbb{C}^{B}
$$

where $x_{2}$ becomes a normalisation constant. The component of $\boldsymbol{x}_{ \pm}$that contributes to the eigenfunction on the first bond of the star graph is its first component, and this is enhanced by a factor approximately $B^{1 / 2}$ in magnitude above the components on all other bonds (which are in fact equal).

We now want to show that $\boldsymbol{x}_{ \pm}$are simple eigenvectors. This follows from the discussion in section 4 . To see it directly we may observe that in extending $\left\{\boldsymbol{x}_{+}, \boldsymbol{x}_{-}\right\}$to an orthogonal basis $\left\{\boldsymbol{x}_{+}, \boldsymbol{x}_{-}, \boldsymbol{x}_{3}, \ldots, \boldsymbol{x}_{B}\right\}$, leads to the first component of $\boldsymbol{x}_{j}, j \geqslant 3$ being zero, and consequently

$$
P(\kappa) \boldsymbol{x}_{j}=\boldsymbol{x}_{j},
$$

for any value of $\kappa$. It then follows that

$$
\mathcal{F}_{B} \boldsymbol{x}_{j}=\lambda_{j} \boldsymbol{x}_{j}
$$

i.e. the vectors $\boldsymbol{x}_{j}$ are eigenvectors of $\mathcal{F}_{B}$, and therefore $\lambda_{j} \in\{ \pm 1, \pm \mathrm{i}\}$. As we have already observed the eigenvalues of $\boldsymbol{x}_{ \pm}$do not belong to the aforementioned set, thus they are simple eigenvectors.

To assist with calculating the entropy of $\boldsymbol{x}_{ \pm}$, we note that

$$
\begin{aligned}
\left\|\boldsymbol{x}_{ \pm}\right\|^{2} & =\left(\left(\cos \kappa \pm\left(B-\sin ^{2} \kappa\right)^{1 / 2}\right)^{2}+B-1\right)\left|x_{2}\right|^{2} \\
& =\left(B-\sin ^{2} \kappa \pm 2 \cos \kappa\left(B-\sin ^{2} \kappa\right)^{1 / 2}+\cos ^{2} \kappa+B-1\right)\left|x_{2}\right|^{2} \\
& =\left(2 B \pm 2 \cos \kappa\left(B-\sin ^{2} \kappa\right)^{1 / 2}-2 \sin ^{2} \kappa\right)\left|x_{2}\right| \\
& =2\left(B \pm \cos \kappa\left(B-\sin ^{2} \kappa\right)^{1 / 2}-\sin ^{2} \kappa\right)\left|x_{2}\right|^{2} \\
& =2\left(B-\sin ^{2} \kappa\right)^{1 / 2}\left(\left(B-\sin ^{2} \kappa\right)^{1 / 2} \pm \cos \kappa\right)\left|x_{2}\right|^{2} .
\end{aligned}
$$

\section{Perturbation of other bond lengths}

In this appendix we calculate eigenvectors of the matrix $F_{j}(\kappa)$ described in (4.5). Recall that this is a rank-one perturbation of the $B \times B$ Fourier transform matrix $\mathcal{F}_{B}$.

We refer to certain quantities defined in section 4: the vectors $\boldsymbol{u}_{j}$ and $\boldsymbol{v}_{j}$, and the vectors $\boldsymbol{w}_{+}, \boldsymbol{w}_{-}, \boldsymbol{w}_{+\mathrm{i}}, \boldsymbol{w}_{-\mathrm{i}}$ defined in (4.8), which are eigenvectors of $\mathcal{F}_{B}$ with eigenvalues respectively: $+1,-1,+\mathrm{i},-\mathrm{i}$. We repeat the important identity

$$
\boldsymbol{e}_{j}=\frac{1}{4}\left(\boldsymbol{w}_{+}-\boldsymbol{w}_{-}-\boldsymbol{w}_{+\mathrm{i}}+\boldsymbol{w}_{-\mathrm{i}}\right)
$$

where $\boldsymbol{e}_{j}$ is the $j$ th standard basis vector.

These calculations properly hold only if $B \geqslant 5$. If $B=3$ or 4 then some details change (the eigenspace $\operatorname{Eig}\left(\mathcal{F}_{B},-\mathrm{i}\right)$ is trivial and the vector $\boldsymbol{w}_{-\mathrm{i}}=0$ ). 


\section{C.1 Eigenvectors of the perturbed matrix}

To save notation we write $K(\kappa):=\mathrm{e}^{2 \mathrm{i} \kappa}-1$.

We look for eigenvectors of $F_{j}(\kappa)$ of the form

$$
\boldsymbol{f}=\alpha_{+} \boldsymbol{w}_{+}+\alpha_{-} \boldsymbol{w}_{-}+\alpha_{+\mathrm{i}} \boldsymbol{w}_{+\mathrm{i}}+\alpha_{-\mathrm{i}} \boldsymbol{w}_{-\mathrm{i}},
$$

which can be re-expressed as

$$
\boldsymbol{f}=\beta_{1} \mathcal{F}_{B} \boldsymbol{u}_{j}+\beta_{2} \mathrm{i} \mathcal{F}_{B} \boldsymbol{v}_{j}+\beta_{3} \boldsymbol{u}_{j}+\beta_{4} \boldsymbol{v}_{j},
$$

with

$$
\left(\begin{array}{l}
\beta_{1} \\
\beta_{2} \\
\beta_{3} \\
\beta_{4}
\end{array}\right)=\left(\begin{array}{cccc}
1 & 1 & 0 & 0 \\
0 & 0 & 1 & 1 \\
1 & -1 & 0 & 0 \\
0 & 0 & -1 & 1
\end{array}\right)\left(\begin{array}{c}
\alpha_{+} \\
\alpha_{-} \\
\alpha_{+\mathrm{i}} \\
\alpha_{-\mathrm{i}}
\end{array}\right)
$$

Since $F_{j}(\kappa)=\left(\mathcal{F}_{B}+K(\kappa) \boldsymbol{e}_{j} \boldsymbol{e}_{j}^{\dagger} \mathcal{F}_{B}\right)$ we use the fact that $\boldsymbol{f}$ is a linear combination of eigenvectors of $\mathcal{F}_{B}$ to write

$$
\begin{aligned}
F_{j}(\kappa) \boldsymbol{f} & =\left(I+K(\kappa) \boldsymbol{e}_{j} \boldsymbol{e}_{j}^{\dagger}\right) \mathcal{F}_{B} \boldsymbol{f} \\
& =\left(I+K(\kappa) \boldsymbol{e}_{j} \boldsymbol{e}_{j}^{\dagger}\right)\left(\alpha_{+} \boldsymbol{w}_{+}-\alpha_{-} \boldsymbol{w}_{-}+\mathrm{i} \alpha_{+\mathrm{i}} \boldsymbol{w}_{+\mathrm{i}}-\mathrm{i} \alpha_{-\mathrm{i}} \boldsymbol{w}_{-\mathrm{i}}\right) .
\end{aligned}
$$

We then use (C.1), and orthogonality of the $\boldsymbol{w}_{i}$ to express

$$
F_{j}(\kappa) \boldsymbol{f}=\left(\alpha_{+} \boldsymbol{w}_{+}-\alpha_{-} \boldsymbol{w}_{-}+\mathrm{i} \alpha_{+\mathrm{i}} \boldsymbol{w}_{+\mathrm{i}}-\mathrm{i} \alpha_{-\mathrm{i}} \boldsymbol{w}_{-\mathrm{i}}\right)+\frac{1}{4} K(\kappa) A\left(\boldsymbol{w}_{+}-\boldsymbol{w}_{-}-\boldsymbol{w}_{+\mathrm{i}}+\boldsymbol{w}_{-\mathrm{i}}\right)
$$

where

$$
A=\frac{1}{4}\left(\alpha_{+}\left\|\boldsymbol{w}_{+}\right\|^{2}+\alpha_{-}\left\|\boldsymbol{w}_{-}\right\|^{2}-\mathrm{i} \alpha_{+\mathrm{i}}\left\|\boldsymbol{w}_{+\mathrm{i}}\right\|^{2}-\mathrm{i} \alpha_{-\mathrm{i}}\left\|\boldsymbol{w}_{-\mathrm{i}}\right\|^{2}\right) .
$$

Equating components in the eigenvalue equation

$$
F_{j}(\kappa) \boldsymbol{f}=\mathrm{e}^{\mathrm{i} \phi} \boldsymbol{f}
$$

we find that the vector $\boldsymbol{\alpha}=\left(\alpha_{+}, \alpha_{-}, \alpha_{+\mathrm{i}}, \alpha_{-\mathrm{i}}\right)^{T}$ satisfies the linear equation system

$$
\left(\begin{array}{cccc}
1+\frac{K(\kappa)}{16}\left\|\boldsymbol{w}_{+}\right\|^{2} & \frac{K(\kappa)}{16}\left\|\boldsymbol{w}_{-}\right\|^{2} & -\frac{\mathrm{i} K(\kappa)}{16}\left\|\boldsymbol{w}_{+\mathrm{i}}\right\|^{2} & -\frac{\mathrm{i} K(\kappa)}{16}\left\|\boldsymbol{w}_{-\mathrm{i}}\right\|^{2} \\
-\frac{K(\kappa)}{16}\left\|\boldsymbol{w}_{+}\right\|^{2} & -1-\frac{K(\kappa)}{16}\left\|\boldsymbol{w}_{-}\right\|^{2} & \frac{\mathrm{i} K(\kappa)}{16}\left\|\boldsymbol{w}_{+\mathrm{i}}\right\|^{2} & \frac{\mathrm{i} K(\kappa)}{16}\left\|\boldsymbol{w}_{-\mathrm{i}}\right\|^{2} \\
-\frac{K(\kappa)}{16}\left\|\boldsymbol{w}_{+}\right\|^{2} & -\frac{K(\kappa)}{16}\left\|\boldsymbol{w}_{-}\right\|^{2} & \mathrm{i}+\frac{\mathrm{i} K(\kappa)}{16}\left\|\boldsymbol{w}_{+\mathrm{i}}\right\|^{2} & \frac{\mathrm{i} K(\kappa)}{16}\left\|\boldsymbol{w}_{-\mathrm{i}}\right\|^{2} \\
\frac{K(\kappa)}{16}\left\|\boldsymbol{w}_{+}\right\|^{2} & \frac{K(\kappa)}{16}\left\|\boldsymbol{w}_{-}\right\|^{2} & -\frac{\mathrm{i} K(\kappa)}{16}\left\|\boldsymbol{w}_{+\mathrm{i}}\right\|^{2} & -\mathrm{i}-\frac{\mathrm{i} K(\kappa)}{16}\left\|\boldsymbol{w}_{-\mathrm{i}}\right\|^{2}
\end{array}\right) \boldsymbol{\alpha}=\mathrm{e}^{\mathrm{i} \phi} \boldsymbol{\alpha}
$$

From (C.4) it follows that

$$
\boldsymbol{\alpha}=\frac{1}{2}\left(\begin{array}{cccc}
1 & 0 & 1 & 0 \\
1 & 0 & -1 & 0 \\
0 & 1 & 0 & -1 \\
0 & 1 & 0 & 1
\end{array}\right) \boldsymbol{\beta}
$$

with $\boldsymbol{\beta}=\left(\beta_{1}, \beta_{2}, \beta_{3}, \beta_{4}\right)^{T}$. We may re-write (C.9) as an equation for $\boldsymbol{\beta}$, using (C.4) and (C.10), as

$$
\left(\begin{array}{cccc}
0 & 0 & 1 & 0 \\
0 & 0 & 0 & -\mathrm{i} \\
1+\frac{K(\kappa)}{16} W_{1} & -\frac{\mathrm{i} K(\kappa)}{16} W_{2} & \frac{K(\kappa)}{16} W_{3} & \frac{\mathrm{i} K(\kappa)}{16} W_{4} \\
\frac{K(\kappa)}{16} W_{1} & -\mathrm{i}-\frac{\mathrm{i} K(\kappa)}{16} W_{2} & \frac{K(\kappa)}{16} W_{3} & \frac{\mathrm{i} K(\kappa)}{16} W_{4}
\end{array}\right) \boldsymbol{\beta}=\mathrm{e}^{\mathrm{i} \phi} \boldsymbol{\beta}
$$


with

$$
\begin{aligned}
& W_{1}:=\left\|\boldsymbol{w}_{+}\right\|^{2}+\left\|\boldsymbol{w}_{-}\right\|^{2} \\
& W_{2}:=\left\|\boldsymbol{w}_{+\mathrm{i}}\right\|^{2}+\left\|\boldsymbol{w}_{-\mathrm{i}}\right\|^{2} \\
& W_{3}:=\left\|\boldsymbol{w}_{+}\right\|^{2}-\left\|\boldsymbol{w}_{-}\right\|^{2} \\
& W_{4}:=\left\|\boldsymbol{w}_{+\mathrm{i}}\right\|^{2}-\left\|\boldsymbol{w}_{-\mathrm{i}}\right\|^{2} .
\end{aligned}
$$

We observe immediately from (C.11) that

$$
\begin{aligned}
\beta_{3} & =\mathrm{e}^{\mathrm{i} \phi} \beta_{1}, \\
-\mathrm{i} \beta_{4} & =\mathrm{e}^{\mathrm{i} \phi} \beta_{2},
\end{aligned}
$$

and, on subtracting the fourth row from the third,

$$
\begin{aligned}
\beta_{1}+\mathrm{i} \beta_{2} & =\mathrm{e}^{\mathrm{i} \phi} \beta_{3}-\mathrm{e}^{\mathrm{i} \phi} \beta_{4} \\
\Rightarrow \quad \mathrm{e}^{-\mathrm{i} \phi} \beta_{3}+\mathrm{e}^{-\mathrm{i} \phi} \beta_{4} & =\mathrm{e}^{\mathrm{i} \phi} \beta_{3}-\mathrm{e}^{\mathrm{i} \phi} \beta_{4}, \quad \text { using (C.13), } \\
\Rightarrow \quad \beta_{3} & =-\mathrm{i} \cot \phi \beta_{4},
\end{aligned}
$$

finally getting

$$
\begin{aligned}
& \beta_{1}=-\mathrm{ie}^{-\mathrm{i} \phi} \cot \phi \beta_{4} \\
& \beta_{2}=-\mathrm{ie}^{-\mathrm{i} \phi} \beta_{4} \\
& \beta_{3}=-\mathrm{i} \cot \phi \beta_{4} .
\end{aligned}
$$

\section{C.2 Eigenvalue equation}

The undetermined constant $\beta_{4}$ will be set by normalisation, but we first turn to the value of $\phi$. For this we will need explicit formulæ for $W_{1}, \ldots W_{4}$.

From (4.8),

$$
\begin{aligned}
\left\|\boldsymbol{w}_{+}\right\|^{2} & =\left(\mathcal{F}_{B} \boldsymbol{u}_{j}+\boldsymbol{u}_{j}\right)^{\dagger}\left(\mathcal{F}_{B} \boldsymbol{u}_{j}+\boldsymbol{u}_{j}\right) \\
& =\left(\boldsymbol{u}_{j}^{\dagger}+\boldsymbol{u}_{j}^{\dagger} \mathcal{F}_{B}^{\dagger}\right)\left(\mathcal{F}_{B} \boldsymbol{u}_{j}+\boldsymbol{u}\right) \\
& =2\|\boldsymbol{u}\|^{2}+\boldsymbol{u}_{j}^{\dagger}\left(\mathcal{F}_{B}^{\dagger}+\mathcal{F}_{B}\right) \boldsymbol{u}_{j},
\end{aligned}
$$

and

$$
\left\|\boldsymbol{w}_{-}\right\|^{2}=2\|\boldsymbol{u}\|^{2}-\boldsymbol{u}_{j}^{\dagger}\left(\mathcal{F}_{B}^{\dagger}+\mathcal{F}_{B}\right) \boldsymbol{u}_{j},
$$

so we immediately see that

$$
W_{1}=\left\|\boldsymbol{w}_{+}\right\|^{2}+\left\|\boldsymbol{w}_{-}\right\|^{2}=4\left\|\boldsymbol{u}_{j}\right\|^{2}=8,
$$

and

$$
W_{3}=\left\|\boldsymbol{w}_{+}\right\|^{2}-\left\|\boldsymbol{w}_{-}\right\|^{2}=2 \boldsymbol{u}_{j}^{\dagger}\left(\mathcal{F}_{B}+\mathcal{F}_{B}^{\dagger}\right) \boldsymbol{u}_{j} .
$$

At this point we use the formula (3.2) for the entries of $\mathcal{F}_{B}$ to deduce that the $n m$ th entry of the matrix $\mathcal{F}_{B}+\mathcal{F}_{B}^{\dagger}$ is

$$
\frac{2}{\sqrt{B}} \cos \left(\frac{2 \pi(n-1)(m-1)}{B}\right),
$$


and the $n$th entry of the vector $\left(\mathcal{F}_{B}+\mathcal{F}_{B}^{\dagger}\right) \boldsymbol{u}_{j}$ is

$$
\begin{array}{r}
\frac{2}{\sqrt{B}} \cos \left(\frac{2 \pi(n-1)(j-1)}{B}\right)+\frac{2}{\sqrt{B}} \cos \left(\frac{2 \pi(n-1)(B+1-j)}{B}\right) \\
=\frac{4}{\sqrt{B}} \cos \left(\frac{2 \pi(n-1)(j-1)}{B}\right)
\end{array}
$$

so that

$$
\begin{aligned}
\boldsymbol{u}_{j}^{\dagger}\left(\mathcal{F}_{B}+\mathcal{F}_{B}^{\dagger}\right) \boldsymbol{u}_{j} & =\frac{4}{\sqrt{B}} \cos \left(\frac{2 \pi(j-1)^{2}}{B}\right)+\frac{4}{\sqrt{B}} \cos \left(\frac{2 \pi(j-1)(B+1-j)}{B}\right) \\
& =\frac{8}{\sqrt{B}} \cos \left(\frac{2 \pi(j-1)^{2}}{B}\right) .
\end{aligned}
$$

This means that, from (C.19),

$$
W_{3}=\frac{16}{\sqrt{B}} \cos \left(\frac{2 \pi(j-1)^{2}}{B}\right) .
$$

In a completely analogous way we find that

$$
\begin{aligned}
& \left\|\boldsymbol{w}_{+\mathrm{i}}\right\|^{2}=2\left\|\boldsymbol{v}_{j}\right\|^{2}+\mathrm{i} \boldsymbol{v}_{j}^{\dagger}\left(\mathcal{F}_{B}^{\dagger}-\mathcal{F}_{B}\right) \boldsymbol{v}_{j}, \\
& \left\|\boldsymbol{w}_{-\mathrm{i}}\right\|^{2}=2\left\|\boldsymbol{v}_{j}\right\|^{2}-\mathrm{i} \boldsymbol{v}_{j}^{\dagger}\left(\mathcal{F}_{B}^{\dagger}-\mathcal{F}_{B}\right) \boldsymbol{v}_{j} .
\end{aligned}
$$

Similar to how we arrived at (C.22),

$$
\boldsymbol{v}_{j}^{\dagger}\left(\mathcal{F}_{B}^{\dagger}-\mathcal{F}_{B}\right) \boldsymbol{v}_{j}=-\frac{8 \mathrm{i}}{\sqrt{B}} \sin \left(\frac{2 \pi(j-1)^{2}}{B}\right) .
$$

Therefore

$$
W_{2}=\left\|\boldsymbol{w}_{+\mathrm{i}}\right\|^{2}+\left\|\boldsymbol{w}_{-\mathrm{i}}\right\|^{2}=4\left\|\boldsymbol{v}_{j}\right\|^{2}=8
$$

and

$$
W_{4}=\left\|\boldsymbol{w}_{+\mathrm{i}}\right\|^{2}-\left\|\boldsymbol{w}_{-\mathrm{i}}\right\|^{2}=\frac{16}{\sqrt{B}} \sin \left(\frac{2 \pi(j-1)^{2}}{B}\right) .
$$

The big matrix in (C.11) becomes now

$$
\left(\begin{array}{cccc}
0 & 0 & 1 & 0 \\
0 & 0 & 0 & -\mathrm{i} \\
1+\frac{K(\kappa)}{2} & -\frac{\mathrm{i} K(\kappa)}{2} & \frac{K(\kappa)}{\sqrt{B}} \cos \left(\frac{2 \pi(j-1)^{2}}{B}\right) & \frac{\mathrm{i} K(\kappa)}{\sqrt{B}} \sin \left(\frac{2 \pi(j-1)^{2}}{B}\right) \\
\frac{K(\kappa)}{2} & -\mathrm{i}-\frac{\mathrm{i} K(\kappa)}{2} & \frac{K(\kappa)}{\sqrt{B}} \cos \left(\frac{2 \pi(j-1)^{2}}{B}\right) & \frac{\mathrm{i} K(\kappa)}{\sqrt{B}} \sin \left(\frac{2 \pi(j-1)^{2}}{B}\right)
\end{array}\right)
$$

To calculate its characteristic polynomial we use profitably the Schur complement formula,

$$
\operatorname{det}\left(\begin{array}{cc}
A & B \\
C & D
\end{array}\right)=\operatorname{det} A \operatorname{det}\left(D-C A^{-1} B\right)
$$


to deduce that

$$
\begin{aligned}
& \operatorname{det}\left(\begin{array}{cccc}
-\lambda & 0 & 1 & 0 \\
0 & -\lambda & 0 & -\mathrm{i} \\
1+\frac{K(\kappa)}{2} & -\frac{\mathrm{i} K(\kappa)}{2} & \frac{K(\kappa)}{\sqrt{B}} \cos \left(\frac{2 \pi(j-1)^{2}}{B}\right)-\lambda & \frac{\mathrm{i} K(\kappa)}{\sqrt{B}} \sin \left(\frac{2 \pi(j-1)^{2}}{B}\right) \\
\frac{K(\kappa)}{2} & -\mathrm{i}-\frac{\mathrm{i} K(\kappa)}{2} & \frac{K(\kappa)}{\sqrt{B}} \cos \left(\frac{2 \pi(j-1)^{2}}{B}\right) & \frac{\mathrm{i} K(\kappa)}{\sqrt{B}} \sin \left(\frac{2 \pi(j-1)^{2}}{B}\right)-\lambda
\end{array}\right) \quad \text { C.30) } \\
&=\lambda^{2} \operatorname{det}\left(\left(\begin{array}{cc}
\frac{K(\kappa)}{\sqrt{B}} \cos \left(\frac{2 \pi(j-1)^{2}}{B}\right)-\lambda & \frac{\mathrm{i} K(\kappa)}{\sqrt{B}} \sin \left(\frac{2 \pi(j-1)^{2}}{B}\right) \\
\frac{K(\kappa)}{\sqrt{B}} \cos \left(\frac{2 \pi(j-1)^{2}}{B}\right) & \frac{\mathrm{i} K(\kappa)}{\sqrt{B}} \sin \left(\frac{2 \pi(j-1)^{2}}{B}\right)-\lambda
\end{array}\right)\right. \\
&\left.+\frac{1}{\lambda}\left(\begin{array}{cc}
1+\frac{K(\kappa)}{2} & -\frac{\mathrm{i} K(\kappa)}{2} \\
\frac{K(\kappa)}{2} & -\mathrm{i}-\frac{\mathrm{i} K(\kappa)}{2}
\end{array}\right)\left(\begin{array}{cc}
1 & 0 \\
0 & -\mathrm{i}
\end{array}\right)\right) \\
&= \operatorname{det}\left(\begin{array}{cc}
\frac{K(\kappa) \lambda}{\sqrt{B}} \cos \left(\frac{2 \pi(j-1)^{2}}{B}\right)+\frac{K(\kappa)}{2}+1-\lambda^{2} & \frac{\mathrm{i} K(\kappa) \lambda}{\sqrt{B}} \sin \left(\frac{2 \pi(j-1)^{2}}{B}\right)-\frac{K(\kappa)}{2} \\
\frac{K(\kappa) \lambda}{\sqrt{B}} \cos \left(\frac{2 \pi(j-1)^{2}}{B}\right)+\frac{K(\kappa)}{2} & \frac{\mathrm{i} K(\kappa) \lambda}{\sqrt{B}} \sin \left(\frac{2 \pi(j-1)^{2}}{B}\right)-\frac{K(\kappa)}{2}-1-\lambda^{2}
\end{array}\right)
\end{aligned}
$$

and, continuing,

$$
\begin{aligned}
= & \left.\frac{K(\kappa) \lambda}{\sqrt{B}} \cos \left(\frac{2 \pi(j-1)^{2}}{B}\right)+\frac{K(\kappa)}{2}+1-\lambda^{2}\right) \\
& \times\left(\frac{\mathrm{i} K(\kappa) \lambda}{\sqrt{B}} \sin \left(\frac{2 \pi(j-1)^{2}}{B}\right)-\frac{K(\kappa)}{2}-1-\lambda^{2}\right) \\
& -\left(\frac{\mathrm{i} K(\kappa) \lambda}{\sqrt{B}} \sin \left(\frac{2 \pi(j-1)^{2}}{B}\right)-\frac{K(\kappa)}{2}\right)\left(\frac{K(\kappa) \lambda}{\sqrt{B}} \cos \left(\frac{2 \pi(j-1)^{2}}{B}\right)+\frac{K(\kappa)}{2}\right) \\
= & -\left(1+\lambda^{2}\right) \frac{K(\kappa) \lambda}{\sqrt{B}} \cos \left(\frac{2 \pi(j-1)^{2}}{B}\right)+\left(1-\lambda^{2}\right) \frac{\mathrm{i} K(\kappa) \lambda}{\sqrt{B}} \sin \left(\frac{2 \pi(j-1)^{2}}{B}\right) \\
& -\frac{K(\kappa)}{2}\left(1+\lambda^{2}\right)-\left(1-\lambda^{2}\right)\left(1+\lambda^{2}\right)-\frac{K(\kappa)}{2}\left(1-\lambda^{2}\right) \\
= & \lambda^{4}-\frac{\lambda^{3} K(\kappa)}{\sqrt{B}} \mathrm{e}^{2 \pi \mathrm{i}(j-1)^{2} / B}-\frac{\lambda K(\kappa)}{\sqrt{B}} \mathrm{e}^{-2 \pi \mathrm{i}(j-1)^{2} / B}-1-K(\kappa) .
\end{aligned}
$$

The eigenphases $\mathrm{e}^{\mathrm{i} \phi}$ appearing in the solutions (C.15) are the zeros $\lambda$ of the polynomial (C.31). Note that if $\kappa=0$, and there is no perturbation, then $K(0)=0$, and the polynomial (C.31) reduces to $\lambda^{4}-1$ with roots $\lambda= \pm 1, \pm \mathrm{i}$, the unperturbed eigenvalues of $\mathcal{F}_{B}$, as to be expected.

\section{C.3 Explicit form of the perturbed eigenvectors}

The eigenvectors we have found are of the form (C.3),

$$
\boldsymbol{f}=\beta_{1} \mathcal{F}_{B} \boldsymbol{u}_{j}+\beta_{2} \mathrm{i} \mathcal{F}_{B} \boldsymbol{v}_{j}+\beta_{3} \boldsymbol{u}_{j}+\beta_{4} \boldsymbol{v}_{j},
$$

where $\beta_{1}, \ldots, \beta_{3}$ satisfy (C.15) depending on $\phi$, the phase of one of the four roots of (C.31). Putting these into (C.32), and re-naming $-\mathrm{i} \beta_{4}=Z$, we get

$$
\begin{aligned}
\boldsymbol{f} & \left.=\beta_{4}\left(-\mathrm{i} \cot \phi\left(\mathrm{e}^{-\mathrm{i} \phi} \mathcal{F}_{B} \boldsymbol{u}_{j}+\boldsymbol{u}_{j}\right)+\mathrm{e}^{-\mathrm{i} \phi} \mathcal{F}_{B} \boldsymbol{v}_{j}+\boldsymbol{v}_{j}\right)\right) \\
& =Z\left(\cot \phi\left(\mathrm{e}^{-\mathrm{i} \phi} \mathcal{F}_{B} \boldsymbol{u}_{j}+\boldsymbol{u}_{j}\right)+\mathrm{i}\left(\mathrm{e}^{-\mathrm{i} \phi} \mathcal{F}_{B} \boldsymbol{v}_{j}+\boldsymbol{v}_{j}\right)\right) .
\end{aligned}
$$


Let

$$
\begin{aligned}
& \boldsymbol{z}_{1}:=\mathrm{e}^{-\mathrm{i} \phi} \mathcal{F}_{B} \boldsymbol{u}_{j}+\boldsymbol{u}_{j} \\
& \boldsymbol{z}_{2}:=\mathrm{e}^{-\mathrm{i} \phi} \mathcal{F}_{B} \boldsymbol{v}_{j}+\boldsymbol{v}_{j},
\end{aligned}
$$

so that

$$
\boldsymbol{f}=Z\left(\cot \phi \boldsymbol{z}_{1}+\mathrm{i} \boldsymbol{z}_{2}\right)
$$

Then

$$
\begin{aligned}
\boldsymbol{z}_{1}^{\dagger} \boldsymbol{z}_{2} & =\left(\mathrm{e}^{\mathrm{i} \phi} \boldsymbol{u}_{j}^{\dagger} \mathcal{F}_{B}^{\dagger}+\boldsymbol{u}_{j}^{\dagger}\right)\left(\mathrm{e}^{-\mathrm{i} \phi} \mathcal{F}_{B} \boldsymbol{v}_{j}+\boldsymbol{v}_{j}\right) \\
& =\boldsymbol{u}_{j}^{\dagger} \boldsymbol{v}_{j}+\mathrm{e}^{\mathrm{i} \phi} \boldsymbol{u}_{j}^{\dagger} \mathcal{F}_{B}^{\dagger} \boldsymbol{v}_{j}+\mathrm{e}^{-\mathrm{i} \phi} \boldsymbol{u}_{j}^{\dagger} \mathcal{F}_{B} \boldsymbol{v}_{j}+\boldsymbol{u}_{j}^{\dagger} \boldsymbol{v}_{j} \\
& =0
\end{aligned}
$$

because $\boldsymbol{u}_{j}^{\dagger} \boldsymbol{v}_{j}=0$ by construction, and since $\boldsymbol{u}_{j}^{\dagger}\left(\mathcal{F}_{B}^{\dagger}\right)^{2}=\boldsymbol{u}_{j}^{\dagger}$ and $\mathcal{F}_{B}^{2} \boldsymbol{v}_{j}=-\boldsymbol{v}_{j}$ we have that

$$
\boldsymbol{u}_{j}^{\dagger} \mathcal{F}_{B} \boldsymbol{v}_{j}=-\boldsymbol{u}_{j}^{\dagger} \mathcal{F}_{B}^{\dagger} \boldsymbol{v}_{j}=-\boldsymbol{u}_{j}^{\dagger}\left(\mathcal{F}_{B}^{\dagger}\right)^{2} \mathcal{F}_{B} \boldsymbol{v}_{j}=-\boldsymbol{u}_{j}^{\dagger} \mathcal{F}_{B} \boldsymbol{v}_{j}
$$

and similar for $\boldsymbol{u}_{j}^{\dagger} \mathcal{F}_{B}^{\dagger} \boldsymbol{v}_{j}$.

A consequence of the orthogonality (C.36) is that

$$
\|\boldsymbol{f}\|^{2}=|Z|^{2}\left(\cot ^{2} \phi\left\|\boldsymbol{z}_{1}\right\|^{2}+\left\|\boldsymbol{z}_{2}\right\|^{2}\right) .
$$

From (C.34),

$$
\left\|\boldsymbol{z}_{1}\right\|^{2}=2\left\|\boldsymbol{u}_{j}\right\|^{2}+\boldsymbol{u}_{j}^{\dagger}\left(\mathrm{e}^{-\mathrm{i} \phi} \mathcal{F}_{B}+\mathrm{e}^{\mathrm{i} \phi} \mathcal{F}_{B}^{\dagger}\right) \boldsymbol{u}_{j} .
$$

Similarly to how (C.22) was obtained, the $m n$th entry of the matrix $\mathrm{e}^{-\mathrm{i} \phi} \mathcal{F}_{B}+\mathrm{e}^{\mathrm{i} \phi} \mathcal{F}_{B}^{\dagger}$ is

$$
\frac{2}{\sqrt{B}} \cos \left(\phi-\frac{2 \pi(n-1)(m-1)}{B}\right)
$$

and the $m$ th entry of the vector $\left(\mathrm{e}^{-\mathrm{i} \phi} \mathcal{F}_{B}+\mathrm{e}^{\mathrm{i} \phi} \mathcal{F}_{B}^{\dagger}\right) \boldsymbol{u}_{j}$ is

$$
\begin{aligned}
\frac{2}{\sqrt{B}} \cos \left(\phi-\frac{2 \pi(j-1)(m-1)}{B}\right)+\frac{2}{\sqrt{B}} & \cos \left(\phi-\frac{2 \pi(B+1-j)(m-1)}{B}\right) \\
& =\frac{4}{\sqrt{B}} \cos \phi \cos \left(\frac{2 \pi(j-1)(m-1)}{B}\right)
\end{aligned}
$$

leading to

$$
\boldsymbol{u}_{j}^{\dagger}\left(\mathrm{e}^{-\mathrm{i} \phi} \mathcal{F}_{B}+\mathrm{e}^{\mathrm{i} \phi} \mathcal{F}_{B}^{\dagger}\right) \boldsymbol{u}_{j}=\frac{8}{\sqrt{B}} \cos \phi \cos \left(\frac{2 \pi(j-1)^{2}}{B}\right)
$$

and so we get from (C.39),

$$
\left\|\boldsymbol{z}_{1}\right\|^{2}=4+\frac{8}{\sqrt{B}} \cos \phi \cos \left(\frac{2 \pi(j-1)^{2}}{B}\right) .
$$

Proceeding similarly,

$$
\left\|\boldsymbol{z}_{2}\right\|^{2}=2\left\|\boldsymbol{v}_{j}\right\|^{2}+\boldsymbol{v}_{j}^{\dagger}\left(\mathrm{e}^{\mathrm{i} \phi} \mathcal{F}_{B}^{\dagger}+\mathrm{e}^{-\mathrm{i} \phi} \mathcal{F}_{B}\right) \boldsymbol{v}_{j},
$$

and we find that

$$
\boldsymbol{v}_{j}^{\dagger}\left(\mathrm{e}^{\mathrm{i} \phi} \mathcal{F}_{B}^{\dagger}+\mathrm{e}^{\mathrm{i} \phi} \mathcal{F}_{B}\right) \boldsymbol{v}_{j}=\frac{8}{\sqrt{B}} \sin \phi \sin \left(\frac{2 \pi(j-1)^{2}}{B}\right),
$$


so that

$$
\left\|\boldsymbol{z}_{2}\right\|^{2}=4+\frac{8}{\sqrt{B}} \sin \phi \sin \left(\frac{2 \pi(j-1)^{2}}{B}\right) .
$$

Putting together (C.46) and (C.43) into (C.38) we get

$$
\begin{aligned}
\|\boldsymbol{f}\|^{2}= & |Z|^{2}\left(4 \cot ^{2} \phi+\frac{8}{\sqrt{B}} \cot ^{2} \phi \cos \phi \cos \left(\frac{2 \pi(j-1)^{2}}{B}\right)\right. \\
& \left.+4+\frac{8}{\sqrt{B}} \sin \phi \sin \left(\frac{2 \pi(j-1)^{2}}{B}\right)\right) \\
= & |Z|^{2} \operatorname{cosec}^{2} \phi\left(4+\frac{8}{\sqrt{B}}\left(\cos ^{3} \phi \cos \left(\frac{2 \pi(j-1)^{2}}{B}\right)+\sin ^{3} \phi \sin \left(\frac{2 \pi(j-1)^{2}}{B}\right)\right)\right) .
\end{aligned}
$$

Let us denote $\boldsymbol{f}=\left(f_{1}, \ldots, f_{B}\right)$. We now turn to explicit calculation of the components squared, $\left|f_{n}\right|^{2}$. It is prudent to consider the cases $n=j, B+2-j$ separately.

Let us note for subsequent calculations, that the $n$th entry of the vector $\mathcal{F}_{B} \boldsymbol{u}_{j}$ is

$$
\frac{1}{\sqrt{B}} \mathrm{e}^{2 \pi \mathrm{i}(n-1)(j-1) / B}+\frac{1}{\sqrt{B}} \mathrm{e}^{2 \pi \mathrm{i}(n-1)(B+1-j) / B}=\frac{2}{\sqrt{B}} \cos \left(\frac{2 \pi(n-1)(j-1)}{B}\right),
$$

and the $n$th entry of the vector $\mathcal{F}_{B} \boldsymbol{v}_{j}$ is

$$
\frac{1}{\sqrt{B}} \mathrm{e}^{2 \pi \mathrm{i}(n-1)(j-1) / B}-\frac{1}{\sqrt{B}} \mathrm{e}^{2 \pi \mathrm{i}(n-1)(B+1-j) / B}=\frac{2 \mathrm{i}}{\sqrt{B}} \sin \left(\frac{2 \pi(n-1)(j-1)}{B}\right) .
$$

Assume that $n \neq j, B+2-j$. Then from (C.33) and the fact that the $n$th components of $\boldsymbol{u}_{j}$ and $\boldsymbol{v}_{j}$ are zero,

$$
\begin{aligned}
f_{n} & =Z\left(\frac{2 \cot \phi}{\sqrt{B}} \mathrm{e}^{-\mathrm{i} \phi} \cos \left(\frac{2 \pi(n-1)(j-1)}{B}\right)-\frac{2}{\sqrt{B}} \mathrm{e}^{-\mathrm{i} \phi} \sin \left(\frac{2 \pi(n-1)(j-1)}{B}\right)\right) \\
& =\frac{2 Z}{\sqrt{B}} \mathrm{e}^{-\mathrm{i} \phi} \operatorname{cosec} \phi \cos \left(\phi+\frac{2 \pi(n-1)(j-1)}{B}\right),
\end{aligned}
$$

and

$$
\left|f_{n}\right|^{2}=\frac{4|Z|^{2}}{B} \operatorname{cosec}^{2} \phi \cos ^{2}\left(\phi+\frac{2 \pi(n-1)(j-1)}{B}\right) .
$$

If $n=j$ or $n=B+2-j$, then $f_{n}$ contains contributions from the vectors $\boldsymbol{u}_{j}$ and $\boldsymbol{v}_{j}$, giving

$$
\begin{aligned}
f_{n}=Z\left(\frac{2 \cot \phi}{\sqrt{B}} \mathrm{e}^{-\mathrm{i} \phi} \cos \left(\frac{2 \pi(n-1)(j-1)}{B}\right)+\cot \phi\right. \\
\left.\quad-\frac{2}{\sqrt{B}} \mathrm{e}^{-\mathrm{i} \phi} \sin \left(\frac{2 \pi(n-1)(j-1)}{B}\right) \pm \mathrm{i}\right) \\
=Z \operatorname{cosec} \phi\left(\frac{2}{\sqrt{B}} \mathrm{e}^{-\mathrm{i} \phi} \cos \left(\phi+\frac{2 \pi(n-1)(j-1)}{B}\right)+\mathrm{e}^{ \pm \mathrm{i} \phi}\right)
\end{aligned}
$$

with the "+" sign taken for $n=j$ and the "-" sign for $n=B+2-j$. It follows that

$$
\begin{aligned}
\left|f_{n}\right|^{2}=|Z|^{2} \operatorname{cosec}^{2} \phi\left(\frac{2}{\sqrt{B}} \mathrm{e}^{-\mathrm{i} \phi}\right. & \left.\cos \left(\phi+\frac{2 \pi(n-1)(j-1)}{B}\right)+\mathrm{e}^{ \pm \mathrm{i} \phi}\right) \\
& \times\left(\frac{2}{\sqrt{B}} \mathrm{e}^{\mathrm{i} \phi} \cos \left(\phi+\frac{2 \pi(n-1)(j-1)}{B}\right)+\mathrm{e}^{\mp \mathrm{i} \phi}\right) .
\end{aligned}
$$


This becomes, for $n=j$,

$$
\left|f_{j}\right|^{2}=|Z|^{2} \operatorname{cosec}^{2} \phi\left(\frac{4}{B} \cos ^{2}\left(\phi+\frac{2 \pi(j-1)^{2}}{B}\right)+\frac{4}{\sqrt{B}} \cos 2 \phi \cos \left(\phi+\frac{2 \pi(j-1)^{2}}{B}\right)+1\right)
$$

and for $n=B+2-j$,

$$
\left|f_{B+2-j}\right|^{2}=|Z|^{2} \operatorname{cosec}^{2} \phi\left(\frac{4}{B} \cos ^{2}\left(\phi-\frac{2 \pi(j-1)^{2}}{B}\right)+\frac{4}{\sqrt{B}} \cos \left(\phi-\frac{2 \pi(j-1)^{2}}{B}\right)+1\right)
$$

It is possible to verify the calculation of the norm $\|\boldsymbol{f}\|^{2},(\mathrm{C} .47)$ by summing over (C.51) with (C.54) and (C.55), but we do not do that here. Moreover, the components (C.51), (C.54) and (C.55), together with (C.47), are sufficient information to calculate the entropy $\mathrm{s}(\boldsymbol{f})$ exactly.

\section{C.4 Entropy calculation}

We now focus on the calculation of the entropy up to terms small in the size $B$ of the matrix.

From (C.47), we have

$$
\|\boldsymbol{f}\|^{2}=4|Z|^{2} \operatorname{cosec}^{2} \phi\left(1+\mathrm{O}\left(B^{-1 / 2}\right)\right) .
$$

We also deduce from (C.54) and (C.55) that

$$
\begin{aligned}
\left|f_{j}\right|^{2} & =|Z|^{2} \operatorname{cosec}^{2} \phi\left(1+\mathrm{O}\left(B^{-1 / 2}\right),\right. \\
\left|f_{B+2-j}\right|^{2} & =|Z|^{2} \operatorname{cosec}^{2} \phi\left(1+\mathrm{O}\left(B^{-1 / 2}\right) .\right.
\end{aligned}
$$

This means that

$$
\frac{\left|f_{j}\right|^{2}}{\|\boldsymbol{f}\|^{2}}=\frac{1}{4}+\mathrm{O}\left(B^{-1 / 2}\right)
$$

and similarly for $f_{B+2-j}$.

If $h(x):=-x \log x$, so that

$$
\mathbf{s}(\boldsymbol{f})=\sum_{n=1}^{B} h\left(\frac{\left|f_{n}\right|}{\|\boldsymbol{f}\|^{2}}\right),
$$

since $h$ is differentiable on $(0,1)$,

$$
h\left(\frac{\left|f_{j}\right|}{\|\boldsymbol{f}\|^{2}}\right)=h\left(\frac{1}{4}\right)+\mathrm{O}\left(B^{-1 / 2}\right)=\frac{1}{2} \log 2+\mathrm{O}\left(B^{-1 / 2}\right),
$$

and $f_{B+2-j}$ contributes the same.

The main contribution to $\mathrm{s}(f)$ comes from the remaining components, with $n \neq j, B+$ $2-j$. Coming from (C.51) we get

$$
\frac{\left|f_{n}\right|^{2}}{\|\boldsymbol{f}\|^{2}}=\frac{1}{B} \cos ^{2}\left(\phi+\frac{2 \pi(n-1)(j-1)}{B}\right)+\mathrm{O}\left(B^{-3 / 2}\right) .
$$


The contribution of each of these components to the entropy is

$$
\begin{array}{r}
h\left(\frac{\left|f_{n}\right|^{2}}{\|\boldsymbol{f}\|^{2}}\right)=\frac{\log B}{B} \cos ^{2}\left(\phi+\frac{2 \pi(n-1)(j-1)}{B}\right) \\
-\frac{1}{B} \cos ^{2}\left(\phi+\frac{2 \pi(n-1)(j-1)}{B}\right) \log \left(\cos ^{2}\left(\phi+\frac{2 \pi(n-1)(j-1)}{B}\right)\right) \\
+\mathrm{O}\left(\frac{\log B}{B^{3 / 2}}\right) .
\end{array}
$$

Adding up the contributions we have

$$
\mathbf{s}(\boldsymbol{f})=S_{1}(\phi, B) \log B-S_{2}(\phi, B)+2 \frac{1}{2} \log 2+\mathrm{O}\left(\frac{\log B}{B^{1 / 2}}\right),
$$

where

$$
S_{1}(\phi, B):=\frac{1}{B} \sum_{\substack{n=1 \\ n \neq j, B+2-j}}^{B} \cos ^{2}\left(\phi+\frac{2 \pi(n-1)(j-1)}{B}\right)
$$

and

$$
S_{2}(\phi, B):=\frac{1}{B} \sum_{\substack{n=1 \\ n \neq j, B+2-j}}^{B} \cos ^{2}\left(\phi+\frac{2 \pi(n-1)(j-1)}{B}\right) \log \left(\cos ^{2}\left(\phi+\frac{2 \pi(n-1)(j-1)}{B}\right)\right) .
$$

The quantities $S_{1}(\phi, B)$ and $S_{2}(\phi, B)$ are Riemann sums and can be estimated up to errors of order $\mathrm{O}\left(B^{-1}\right)$ by integrals: for $S_{1}$,

$$
\begin{aligned}
S_{1}(\phi, B) & =\int_{0}^{1} \cos ^{2}(\phi+2 \pi(j-1) x) \mathrm{d} x+\mathrm{O}\left(B^{-1}\right) \\
& =\frac{1}{2 \pi} \int_{0}^{2 \pi} \cos ^{2}(\phi+y) \mathrm{d} y+\mathrm{O}\left(B^{-1}\right) \\
& =\frac{1}{2}+\mathrm{O}\left(B^{-1}\right),
\end{aligned}
$$

and

$$
\begin{aligned}
S_{2}(\phi, B) & =\int_{0}^{1} \cos ^{2}(\phi+2 \pi(j-1) x) \log \left(\cos ^{2}(\phi+2 \pi(j-1) x)\right) \mathrm{d} x+\mathrm{O}\left(B^{-1}\right) \\
& =\frac{1}{2 \pi} \int_{0}^{2 \pi} \cos ^{2} y \log \left(\cos ^{2} y\right) \mathrm{d} y+\mathrm{O}\left(B^{-1}\right) \\
& =\frac{1}{2 \pi} \int_{0}^{2 \pi}\left(\frac{1}{2}+\frac{1}{2} \cos 2 y\right) \log \left(\frac{1}{2}+\frac{1}{2} \cos 2 y\right) \mathrm{d} y+\mathrm{O}\left(B^{-1}\right)
\end{aligned}
$$

The integral in (C.67) is of the type considered in appendix D. From the evaluation contained therein, we find that

$$
S_{2}(\phi, B)=-\log 2+\frac{1}{2}+\mathrm{O}\left(B^{-1}\right) .
$$

Finally, putting (C.68) and (C.66) into (C.63), the entropy of $\boldsymbol{f}$ is

$$
\mathbf{s}(\boldsymbol{f})=\frac{1}{2} \log B+2 \log 2-\frac{1}{2}+\mathrm{O}\left(\frac{\log B}{B^{1 / 2}}\right) .
$$




\section{Evaluation of an integral}

In this appendix we evaluate the integral

$$
I(\alpha, \beta, a, b):=\int_{0}^{2 \pi}(\alpha+\beta \cos x) \log (a+b \cos x) \mathrm{d} x, \quad a>0,|b|<a .
$$

In the application we have in mind $\alpha=a$ and $\beta=b$, but it is no extra effort to consider the more general form.

For simplicity we initially suppose that $a=1+t^{2}, b=2 t$ for some $t$ with $|t|<1$. Then

$$
\begin{aligned}
\log \left(1+t^{2}+2 t \cos x\right) & =\log \left(\left(1+t \mathrm{e}^{\mathrm{i} x}\right)\left(1+t \mathrm{e}^{-\mathrm{i} x}\right)\right) \\
& =\log \left(1+t \mathrm{e}^{\mathrm{i} x}\right)+\log \left(1+t \mathrm{e}^{-\mathrm{i} x}\right) .
\end{aligned}
$$

So

$$
I\left(\alpha, \beta, 1+t^{2}, 2 t\right)=\int_{0}^{2 \pi}\left(\alpha+\frac{\beta}{2}\left(\mathrm{e}^{\mathrm{i} x}+\mathrm{e}^{-\mathrm{i} x}\right)\right)\left(\log \left(1+t \mathrm{e}^{\mathrm{i} x}\right)+\log \left(1+t \mathrm{e}^{-\mathrm{i} x}\right)\right) \mathrm{d} x .
$$

We write the logarithmic terms as absolutely uniformly (in $x$ ) convergent series:

$$
\log \left(1+t \mathrm{e}^{ \pm \mathrm{i} x}\right)=-\sum_{n=1}^{\infty} \frac{(-t)^{n} \mathrm{e}^{ \pm \mathrm{i} n x}}{n}
$$

Inserting (D.5) into (D.4) we find that only the $n=1$ terms of the summations contribute and we get

$$
I\left(\alpha, \beta, 1+t^{2}, 2 t\right)=2 \pi \beta t .
$$

If $a$ and $b$ happen not to be of the special form above, we can write

$$
\begin{array}{r}
\log (a+b \cos x)=\log \left(\frac{2 a}{b^{2}}\left(a-\sqrt{a^{2}-b^{2}}\right)+\frac{2}{b}\left(a-\sqrt{a^{2}-b^{2}}\right) \cos x\right) \\
-\log \left(\frac{2}{b^{2}}\left(a-\sqrt{a^{2}-b^{2}}\right)\right),
\end{array}
$$

where the $x$-dependent term is of the form (D.2), with

$$
t=\frac{1}{b}\left(a-\sqrt{a^{2}-b^{2}}\right)
$$

and

$$
\begin{aligned}
1+t^{2} & =1+\frac{1}{b^{2}}\left(a-\sqrt{a^{2}-b^{2}}\right)^{2} \\
& =\frac{2 a}{b^{2}}\left(a-\sqrt{a^{2}-b^{2}}\right) .
\end{aligned}
$$

Thus,

$$
I(\alpha, \beta, a, b)=I\left(\alpha, \beta, 1+t^{2}, 2 t\right)-2 \pi \alpha \log \left(\frac{2}{b^{2}}\left(a-\sqrt{a^{2}-b^{2}}\right)\right)
$$


with $t$ given by (D.8). Noting that

$$
\begin{aligned}
-\log \left(\frac{2}{b^{2}}\left(a-\sqrt{a^{2}-b^{2}}\right)\right) & =\log \left(\frac{b^{2}}{2} \frac{1}{a-\sqrt{a^{2}-b^{2}}}\right) \\
& =\log \left(\frac{a+\sqrt{a^{2}-b^{2}}}{2}\right),
\end{aligned}
$$

we get that

$$
I(\alpha, \beta, a, b)=2 \pi \alpha \log \left(\frac{a+\sqrt{a^{2}-b^{2}}}{2}\right)+2 \pi \beta\left(\frac{a-\sqrt{a^{2}-b^{2}}}{b}\right) .
$$

The case $\alpha=1, \beta=0$ of this integral is formula 4.224.9 of [56].

Finally, if $\alpha=a$ and $\beta=b$, then the integrand (D.1) is bounded and we may increase $b$ to $a$ giving

$$
I(a, a, a, a)=2 \pi a\left(1+\log \left(\frac{a}{2}\right)\right) .
$$

\section{References}

[1] E. J. Heller (1984) "Bound-state eigenfunctions of classically chaotic Hamiltonian systems: scars of periodic orbits," Phys. Rev. Lett. 53, pp. 1515-1518.

[2] E. B. Bogomolny (1988) "Smoothed wave functions of chaotic quantum systems," Physica 31D, pp. 169-189.

[3] O. Agam and S. Fishman (1994) "Semiclassical criterion for scars in wave functions of chaotic systems," Phys. Rev. Lett. 73, pp. 806-809.

[4] M. V. Berry (1989) "Quantum scars of classical closed orbits in phase space," Proc. R. Soc. London, Ser. A 423, pp. 219-231.

[5] L. Kaplan (1999) "Scars in quantum chaotic wavefunctions," Nonlinearity 12, pp. R1R40.

[6] J. P. Keating and S. D. Prado (2001) "Orbit bifurcations and the scarring of wavefunctions," Proc. R. Soc. London, Ser. A 457, pp. 1855-1872.

[7] H. Schanz and T. Kottos (2003) "Scars on quantum networks ignore the Lyapunov exponent," Phys. Rev. Lett. 90, art. no. 234101.

[8] F. Faure, S. Nonnenmacher, and S. de Bièvre (2003) "Scarred eigenstates for quantum cat maps of minimal periods," Commun. Math. Phys. 239, pp. 449-492.

[9] Y. Colin de Verdière (2015) "Semi-classical measures on quantum graphs and the Gauß map of the determinant manifold," Ann. Henri Poincaré 16, pp. 347-364.

[10] G. Berkolaiko, J. P. Keating, and B. Winn (2004) "No quantum ergodicity for star graphs," Commun. Math. Phys. 250, pp. 259-285.

[11] D. Kelmer (2007) "Scarring on invariant manifolds for perturbed quantized hyperbolic toral automorphisms," Commun. Math. Phys. 276, pp. 381-395. 
[12] N. Anantharaman and S. Nonnenmacher (2007) "Entropy of semiclassical measures of the Walsh-quantized baker's map," Ann. Henri Poincaré 8, pp. 37-74.

[13] A. Hassell (2010) "Ergodic billiards that are not quantum unique ergodic," Ann. of Math. 171, pp. 605-618. With an appendix by A. Hassell and L. Hillairet.

[14] E. Lindenstrauss (2006) "Invariant measures and arithmetic quantum unique ergodicity," Ann. of Math. 163, pp. 165-219.

[15] S. Brooks and E. Lindenstrauss (2010) "Graph eigenfunctions and quantum unique ergodicity," C. R. Math. Acad. Sci. Paris.

[16] N. Anantharaman (2008) "Entropy and the localization of eigenfunctions," Ann. of Math. 168, pp. 435-475.

[17] N. Anantharaman and S. Nonnenmacher (2007) "Half-delocalization of eigenfunctions for the Laplacian on an Anosov manifold," Ann. Inst. Fourier 57, pp. 2465-2523.

[18] G. Rivière (2010) "Entropy of semiclassical measures for nonpositively curved surfaces," Ann. Henri Poincaré 11, pp. 1085-1116.

[19] F. Faure and S. Nonnenmacher (2004) "On the maximal scarring for quantum cat map eigenstates," Commun. Math. Phys. 245, pp. 201-214.

[20] T. Kottos and U. Smilansky (1997) "Quantum chaos on graphs," Phys. Rev. Lett. 79, pp. 4794-4797.

[21] T. Kottos and U. Smilansky (1999) "Periodic orbit theory and spectral statistics for quantum graphs," Ann. Phys. 274, pp. 76-124.

[22] G. Berkolaiko and J. P. Keating (1999) "Two-point spectral correlations for star graphs," J. Phys. A 32, pp. 7827-7841.

[23] T. Kottos and H. Schanz (2001) "Quantum graphs: a model for quantum chaos," Physica 9E, pp. 523-530.

[24] F. Barra and P. Gaspard (2000) "On the level spacing distribution in quantum graphs," J. Stat. Phys. 101, pp. 283-319.

[25] G. Berkolaiko, E. B. Bogomolny, and J. P. Keating (2001) "Star graphs and Šeba billiards," J. Phys. A 34, pp. 335-350.

[26] G. Berkolaiko, H. Schanz, and R. S. Whitney (2002) "The leading off-diagonal correction to the form factor of large graphs," Phys. Rev. Lett. 82, art. no. 104101.

[27] J. Bolte and J. Harrison (2003) "The spin contribution to the form factor of quantum graphs," J. Phys. A 36, pp. L433-L440.

[28] G. Berkolaiko, H. Schanz, and R. S. Whitney (2003) "Form factor for a family of quantum graphs: an expansion to third order," J. Phys. A 36, pp. 8373-8392.

[29] G. Berkolaiko (2004) "Form factor for large quantum graphs: evaluating orbits with time-reversal," Waves Random Media 14, pp. S7-S27. 
[30] S. Gnutzmann and A. Altland (2005) "Spectral correlations of individual quantum graphs," Phys. Rev. E 72, art. no. 056215.

[31] S. Gnutzmann, J. P. Keating, and F. Piotet (2008) "Quantum ergodicity on graphs," Phys. Rev. Lett. 101, art. no. 264102.

[32] M. Brammall and B. Winn (2016) "Quantum ergodicity for quantum graphs without back-scattering," Ann. Henri Poincaré 17, pp. 1353-1382.

[33] L. Kameni and R. Schubert "Entropy of eigenfunctions on quantum graphs." Preprint arXiv: 1405.5871.

[34] G. Berkolaiko, J. P. Keating, and B. Winn (2003) "Intermediate wavefunction statistics," Phys. Rev. Lett 91, art. no. 134103.

[35] J. M. Harrison, U. Smilansky, and B. Winn (2007) "Quantum graphs where backscattering is prohibited," J. Phys. A 40, pp. 14181-14193.

[36] O. Bohigas, M.-J. Giannoni, and C. Schmit (1984) "Characterization of chaotic quantum spectra and universality of level fluctuation laws," Phys. Rev. Lett. 52, pp. 1-4.

[37] G. Casati, F. Valz-Griz, and I. Guarneri (1980) "On the connection between the quantization of nonintegrable systems and statistical theory of spectra," Lett. Nuovo Cimento 28, pp. 279-282.

[38] J. P. Keating (1991) "The cat maps: quantum mechanics and classical motion," Nonlinearity 4, pp. 309-341.

[39] S. Gnutzmann and U. Smilansky (2006) "Quantum graphs: Applications to quantum chaos and universal spectral statistics," Adv. Phys. 55, pp. 527-625.

[40] G. Berkolaiko and P. Kuchment Introduction to Quantum Graphs vol. 186 of Mathematical Surveys and Monographs. American Mathematical Society 2013.

[41] P. Kuchment (2004) "Quantum graphs. I. Some basic structures," Waves Random Media 14, pp. S107-S128. Special section on quantum graphs.

[42] J. T. Chalker and P. Coddington (1988) "Percolation, quantum tunnelling and the integer Hall effect," J. Phys. C 21, pp. 2665-2679.

[43] H. Schanz and U. Smilansky (2000) "Spectral statistics for quantum graphs: periodic orbits and combinatorics," Phil. Mag. B 80, pp. 1999-2021. (Proceedings of the Australian summer school on quantum chaos and mesoscopics).

[44] G. Tanner (2001) "Unitary stochastic matrix ensembles and spectral statistics," J. Phys. A 34, pp. 8485-8500.

[45] G. Berkolaiko "Two constructions of quantum graphs and two types of spectral statistics," in Analysis on graphs and its applications vol. 77 of Proc. Sympos. Pure Math. pp. 315-329 Amer. Math. Soc., Providence, RI 2008.

[46] R. Carlson (1999) "Inverse eigenvalue problems on directed graphs," Trans. Amer. Math. Soc. 351, pp. 4069-4088. 
[47] H. Maassen and J. B. M. Uffink (1988) "Generalized entropic uncertainty relations," Phys. Rev. Lett. 60, pp. 1103-1106.

[48] A. E. Rastegin (2017) "Rényi and Tsallis entropies related to eigenfunctions of quantum graphs," J. Phys. A 50, art. no. 215204.

[49] L. Auslander and R. Tolimieri (1979) "Is computing with the finite Fourier transform pure or applied mathematics?," Bull. Amer. Math. Soc. 1, pp. 847-897.

[50] J. H. McClellan and T. W. Parks (1972) "Eigenvalue and eigenvector decomposition of the discrete Fourier transform," IEEE Trans. Audio Electroacoust. AU-20, pp. 6674 .

[51] K. Kraus (1987) "Complementary observables and uncertainty relations," Phys. Rev. D 35, pp. 3070-3075.

[52] S. Albeverio, F. Gesztesy, R. Høegh-Krohn, and H. Holden Solvable models in quantum mechanics. AMS Chelsea Publishing, Providence, RI 2nd ed. 2005. With an appendix by Pavel Exner.

[53] E. B. Bogomolny (2017) "Modification of the Porter-Thomas distribution by rank-one interaction," Phys. Rev. Lett. 118, pp. 022501-1-5.

[54] Y. Colin de Verdière (1982) "Pseudo-laplaciens. I," Ann. Inst. Fourier (Grenoble) 32, pp. xiii, 275-286.

[55] E. Bogomolny, R. Dubertrand, and C. Schmit (2009) "Spectral statistics of a pseudointegrable map: the general case," Nonlinearity 22, pp. 2101-2126.

[56] I. S. Gradshteyn and I. M. Ryzhik Table of integrals, series, and products. Elsevier/Academic Press, Amsterdam 7th ed. 2007. 